\title{
Quantum critical thermal transport in the unitary Fermi gas
}

\author{
Bernhard Frank, ${ }^{1}$ Wilhelm Zwerger, ${ }^{2}$ and Tilman Enss $\oplus^{3}$ \\ ${ }^{1}$ Max-Planck-Institut für Physik komplexer Systeme, 01187 Dresden, Germany \\ ${ }^{2}$ Technische Universität München, Physik Department, James-Franck-Strasse, 85748 Garching, Germany \\ ${ }^{3}$ Institut für Theoretische Physik, Universität Heidelberg, 69120 Heidelberg, Germany
}

(Received 23 March 2020; accepted 19 May 2020; published 8 June 2020)

\begin{abstract}
Strongly correlated systems are often associated with an underlying quantum critical point which governs their behavior in the finite-temperature phase diagram. Their thermodynamical and transport properties arise from critical fluctuations and follow universal scaling laws. Here, we develop a microscopic theory of thermal transport in the quantum critical regime expressed in terms of a thermal sum rule and an effective scattering time. We explicitly compute the characteristic scaling functions in a quantum critical model system, the unitary Fermi gas. Moreover, we derive an exact thermal sum rule for heat and energy currents and evaluate it numerically using the nonperturbative Luttinger-Ward approach. For the thermal scattering times we find a simple quantum critical scaling form. Together, the sum rule and the scattering time determine the heat conductivity, thermal diffusivity, Prandtl number, and sound diffusivity from high temperatures down into the quantum critical regime. The results provide a quantitative description of recent sound attenuation measurements in ultracold Fermi gases.
\end{abstract}

DOI: 10.1103/PhysRevResearch.2.023301

\section{INTRODUCTION}

Thermal transport caused by temperature gradients is ubiquitous in nature and typically occurs in a diffusive manner. A calculation of the corresponding thermal conductivity $\kappa$ and the associated thermal diffusion constant $D_{T}=\kappa / c_{p}$ is often based on a kinetic theory description like the Boltzmann equation. This works well, e.g., in metals at low temperature and allows one to understand the origin of universal laws like the Lorenz ratio $L=\kappa / \sigma T \rightarrow L_{0}=\pi^{2} k_{B}^{2} / 3 e^{2}$ between the thermal and the electrical conductivity $\sigma$ as predicted by Wiedemann and Franz. In strongly correlated systems, sometimes called bad metals [1], the underlying Fermi-liquid description does not apply, however, and $L$ deviates substantially from its ideal value $L_{0}$ [2,3]. Developing a microscopic theory for thermal transport in non-Fermi liquids has been a major challenge for many years. It has been approached using a number of different techniques like the memory function formalism [4]. In a number of cases, a possible and phenomenologically often successful strategy to describe transport in the absence of well-defined quasiparticles is to assume the existence of an underlying quantum critical point (QCP) [5]. Transport in the quantum critical regime (QCR) above the QCP may then be analyzed in terms of critical fluctuations where decay and scattering rates typically scale linearly with temperature according to a Planckian law $\tau^{-1} \propto$ $k_{B} T / \hbar$ [5-7], a behavior which has been observed recently in the thermal diffusivity of near optimally doped cuprates

Published by the American Physical Society under the terms of the Creative Commons Attribution 4.0 International license. Further distribution of this work must maintain attribution to the author(s) and the published article's title, journal citation, and DOI. above the superconducting transition [8]. The aim of this work is to develop a microscopic theory for thermal transport in a much simpler system with a quantum critical point, namely, the unitary Fermi gas (UFG) $[9,10]$. This system has a QCP at zero density which is both scale and conformally invariant [11-14]. In the quantum critical regime above this point, the thermal wavelength $\lambda_{T}=h / \sqrt{2 \pi m T}$ (we set $k_{B}=$ 1 throughout the paper) and the characteristic time $\hbar / T$ are the only relevant length scales and timescales. Correlation functions involving observables that do not develop anomalous dimensions associated with details of the interaction at short distances thus obey simple scaling laws [10]. This applies, for instance, to the shear viscosity $\eta$ and the related ratio $\eta / s$ with the entropy density $s$, which turns out to be not far above the well-known Kovtun-Son-Starinets bound [15-18]. Similarly, the spin diffusion constant $D_{s}$ exhibits the quantum critical scaling behavior, and a minimum value $D_{s} \simeq 1.3 \hbar / \mathrm{m}$ has been measured and determined theoretically [19-22].

Very recently, experiments with dilute ultracold atomic gases have realized homogeneous Fermi gases [24,25] and opened the possibility to access local thermal transport via the diffusive spreading of density and thermal wave packets propagating in a sufficiently large box [23,26-29]. These experiments are considerably more sensitive than previous global transport measurements from trap collective modes. For instance, the measurements of the hydrodynamic sound dispersion $\omega_{q}=c_{s} q-i D_{\text {sound }} q^{2} / 2+\ldots$ in a homogeneous unitary Fermi gas of ${ }^{6} \mathrm{Li}$ atoms at MIT [23] provide both the speed of sound $c_{s}$ and the sound diffusivity $D_{\text {sound }}=$ $(4 / 3) D_{\eta}+\left(c_{p} / c_{V}-1\right) D_{T}$ [30]. Knowledge of the kinematic viscosity $D_{\eta}=\eta /(m n)[16,17]$ and the Landau-Placzek ratio $\mathrm{LP}=c_{p} / c_{V}-1$ (Fig. 7 below) gives then access to the thermal diffusivity $D_{T}$ in the quantum degenerate gas (see Fig. 1). Theoretical results for thermal transport are so far available only at high temperature from the virial expansion 


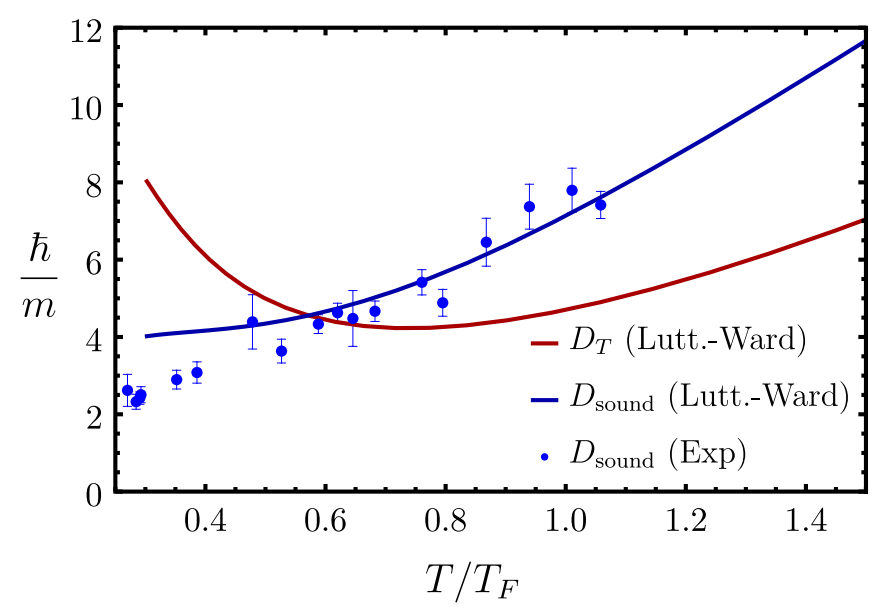

FIG. 1. Thermal diffusivity $D_{T}$ (red) and sound diffusivity $D_{\text {sound }}$ (blue) vs temperature $T / T_{F}$ in the quantum critical regime of the unitary Fermi gas. Theoretical results from Luttinger-Ward calculations are shown in comparison with sound diffusion measurements [23].

[31]. It is the goal of this work to compute thermal transport at low temperature and in particular in the quantum critical regime.

In the following, we compute thermal transport in the quantum critical region of the unitary Fermi gas based on a decomposition of the thermal conductivity as a product

$$
\kappa T=\chi_{q q}^{T} \tau_{\kappa}
$$

of a nontrivial, thermodynamic sum rule $\chi_{q q}^{T}$ for the heat current response and a thermal scattering time $\tau_{\kappa}$ which can formally be derived within a memory function approach (cf. Sec. II). We show that both factors of this decomposition can be described by universal scaling forms which smoothly connect the quantum critical to the high-temperature regime, where a virial expansion for the thermodynamic properties and a Boltzmann equation for the associated scattering time is applicable. In Sec. III we derive an exact expression for the thermal sum rule $\chi_{q q}^{T}$ in terms of Green's functions with the help of Ward identities for energy and particle-number conservation. Based on nonperturbative results for the Green's functions from a fully self-consistent Luttinger-Ward computation [32-34], we evaluate $\chi_{q q}^{T}$ numerically. We find a strong enhancement of spectral weight in the quantum critical regime compared to the noninteracting gas which reaches two orders of magnitude in the quantum critical regime just above the superfluid transition. In Sec. IV we compute the thermal scattering time $\tau_{\kappa}$ of order $T / \hbar$ using a large- $N$ expansion. Quite unexpectedly, the time $\tau_{\kappa}$ extrapolates in a simple manner from the Boltzmann gas limit down into the quantum critical regime. In Sec. V, we combine the results for the sum rule with the scattering times in Eq. (1) to predict the thermal transport coefficient $\kappa$, the diffusivity $D_{T}$ shown in Fig. 1, and the Prandtl number Pr. In particular, we find good agreement with the experimentally observed values in the quantum critical regime. We conclude with a discussion in Sec. VI.

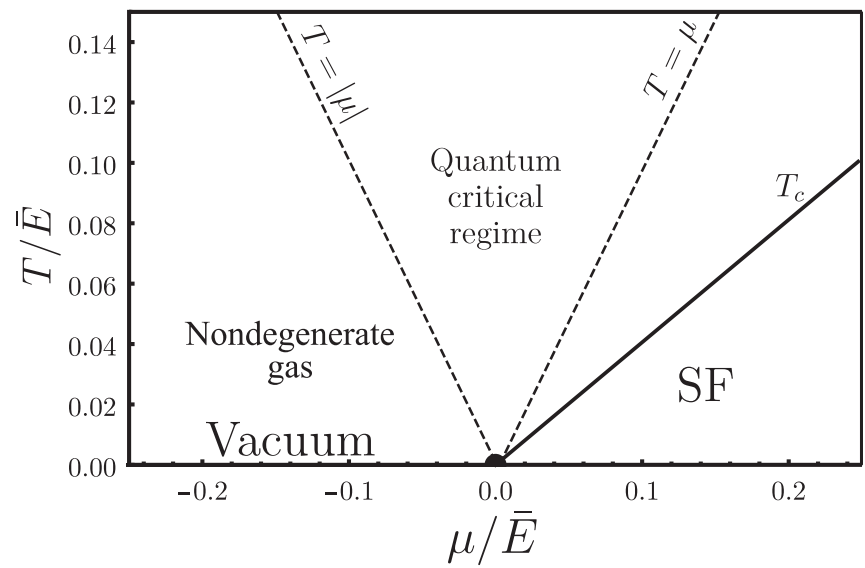

FIG. 2. Phase diagram of the spin-balanced, unitary Fermi gas at finite temperatures [36]. The QCP at $T=0, \mu=0$ is the starting point for the phase boundary of the homogeneous superfluid at $T_{c}=0.4 \mu$ (solid line). The dashed lines mark the crossover to the quantum critical regime above the QCP.

\section{QUANTUM CRITICAL THERMAL TRANSPORT}

In this section we first define the quantum critical regime of the unitary Fermi gas in Sec. II A, and discuss the crossover to classical critical behavior close to the finite-temperature superfluid transition. In Sec. II B, we discuss the formal structure of how to compute thermal transport in linear response from the Kubo formula and its evaluation within the memory function formalism.

\section{A. Quantum critical regime}

Dilute ultracold Fermi gases interact via a short-range attractive interaction between different spin components [35]. At low temperature, atoms scatter predominantly in the $s$ wave channel with scattering amplitude $f(k)=-1 /\left(a^{-1}+\right.$ $i k)$, which is fully characterized by the $s$-wave scattering length $a$. Here, we focus on the unitary limit $1 / a=0$ that gives rise to a strongly interacting system as the standard perturbative expansion in a small gas parameter $n|a|^{3} \ll 1$ breaks down. The phase diagram shown in Fig. 2 exhibits a quantum critical point at vanishing chemical potential and temperature $\mu=T=0$, which separates the vacuum state at $\mu<0$ from a homogeneous superfluid (SF) state at $\mu>$ $0[10,11,36]$. Here, all energies are expressed in terms of $\bar{E}$, which is of the order of the van der Waals energy that sets the cutoff scale beyond which details of the interaction potential start to matter. The universal description based on the model Hamiltonian (10) below is thus applicable only for $\mu, T \ll \bar{E}$. In the absence of a finite effective Zeeman field $h=\left(\mu_{\uparrow}-\mu_{\downarrow}\right) / 2$ which may lead to nontrivial phases with a finite spin population imbalance [33,34], the phase diagram is characterized by a single dimensionless parameter $\beta \mu$. The superfluid state remains stable for temperatures below the critical curve $T_{c} \simeq 0.4 \mu$ or equivalently $(\beta \mu)_{c} \simeq 2.5$ [37]. Instead, for high temperature or small fugacity $z=e^{\beta \mu} \ll 1$ the system forms a dilute, nondegenerate gas which can be described in terms of the virial expansion. Increasing the fugacity $z$ to values of order unity one enters the QCR, as 
shown in Fig. 2. In this regime, both thermodynamic and transport properties follow universal scaling laws associated with the zero density fixed point at $T=\mu=0$, with $\beta \mu$ as the single relevant scaling variable [11].

The quantum critical scaling is replaced by the one characteristic for a classical $d=3 \mathrm{XY}$ model close to the superfluid phase transition at $\mu_{c}(T) \simeq 2.5 T$. This crossover occurs when the Gaussian correlation length $\xi_{G}$ of the quantum model, defined by the quadratic term $1 / \xi_{G}^{2}=2 m\left(\mu-\mu_{c}\right) / \hbar^{2}$ in the Ginzburg-Landau free energy, becomes of the same order as the characteristic length $\xi_{1}$. The length $\xi_{1} \simeq 1 / u_{0}$ is associated with the coefficient $u_{0}$ of the quartic term $\left(u_{0} / 4 !\right)\left[\phi_{1}^{2}(\mathbf{x})+\phi_{2}^{2}(\mathbf{x})\right]^{2}$ of the classical $\phi^{4}$ theory for a complex scalar field $\phi(\mathbf{x})=\phi_{1}(\mathbf{x})+i \phi_{2}(\mathbf{x})$ that depends only on space. This term may in principle be derived from the usual complex order parameter $\psi(\mathbf{x}, \tau)$ for the superfluid transition by integrating out all nonzero Matsubara frequencies $\Omega_{n} \neq 0$. In explicit form, this has been worked out for a generalization of the proper $N=2$ component model of a weakly interacting Bose gas to a large number $N$, which yields $u_{0}^{\mathrm{BEC}}(a)=96 \pi^{2} a / \lambda_{T}^{2}$ in the $N=\infty$ limit [38]. In the case of the unitary Fermi gas at $1 / a=0$, simple dimensional analysis requires that $u_{0} \simeq 1 / \lambda_{T}$, however, the value of the numerical prefactor is unknown. Qualitatively, the crossover condition $\xi_{1} \simeq \xi_{G}$ thus gives the simple relation $\mu-\mu_{c}(T) \simeq$ $k_{B} T$, which entails a Ginzburg parameter of order unity and a very large Ginzburg region that extends up to about $2 T_{c}$ as discussed by Debelhoir and Dupuis [39].

In the vicinity of the superfluid transition, the quantum critical scaling of dynamical quantities is replaced by the classical dynamical scaling. In particular, the thermal conductivity of the UFG is described within model F [40] with dynamical critical exponent $z=\frac{3}{2}$ for the superfluid transition in the universality class of the $d=3 \mathrm{XY}$ model. As shown by Ferrell et al. [41], this implies a divergent thermal conductivity

$$
\kappa \sim\left(T-T_{c}\right)^{-v / 2} \sim\left(T-T_{c}\right)^{-1 / 3},
$$

as $T \rightarrow T_{c}^{+}$, which diverges with an exponent close to $\frac{1}{3}$ since $v \approx \frac{2}{3}$.

\section{B. Linear response and memory function formalism for the thermal conductivity}

A formally exact expression which in principle allows to calculate transport coefficients for an arbitrary form of the underlying Hamiltonian is based on linear response theory and the resulting Kubo formula. In the special case of the thermal conductivity at external momentum $\mathbf{q}=0$, it is convenient to consider the heat current density $\mathbf{j}^{q}$ [42], which is defined as the energy current $\mathbf{j}^{E}$ at constant particle number, i.e., with the enthalpy per particle $w / n$ times the number current density $\mathbf{j}$ subtracted:

$$
\mathbf{j}^{q}=\mathbf{j}^{E}-(w / n) \mathbf{j}=\mathbf{j}^{E}-(\mu+T \tilde{s}) \mathbf{j} .
$$

Here, we have used the Gibbs-Duhem relation $w=\varepsilon+p=$ $\mu n+T s$ and defined the entropy per particle $\tilde{s}=s / n$. In standard hydrodynamic terms this corresponds to the definition of the thermal conductivity via Fourier's law $\mathbf{j}^{E}=-\kappa \nabla T$ in the absence of a particle current. Microscopically, the effect of a weak temperature gradient is encoded in the equilibrium retarded heat current response function from linear response theory,

$$
\chi_{q q}(\omega)=\frac{i}{\hbar} \int_{0}^{\infty} d t e^{i \omega t} \int d^{3} x\left\langle\left[\hat{\boldsymbol{J}}^{q}(\mathbf{x}, t), \hat{\boldsymbol{J}}^{q}(\mathbf{0}, 0)\right]\right\rangle_{\mathrm{eq}},
$$

where we suppress the argument $\mathbf{q}=0$ from now on. The retarded commutator in Eq. (4) defines a positive and even spectral representation of the dynamic thermal conductivity

$$
\kappa(\omega) T=\frac{\operatorname{Im} \chi_{q q}(\omega)}{\omega}, \quad \kappa=\lim _{\omega \rightarrow 0} \kappa(\omega) .
$$

Since a fully microscopic evaluation of the frequencydependent response function in a strongly interacting manybody system is impossible, it is necessary to reduce the problem by restricting attention to the dc response and a simplified procedure to evaluate the characteristic timescale $\tau_{\kappa}$ defined in Eq. (1). Such a procedure is provided by the memory function formalism. It has been used to determine the dynamical charge conductivity of simple metals some time ago by Götze and Wölfle [43] and it provides a systematic and unified description for the derivation of hydrodynamic equations of motion in fluids (see, e.g., the textbook by Forster [30]). More recently, the method has been applied successfully to calculate transport coefficients in systems without well-defined quasiparticles $[4,6,44]$. It is based on a formal expression for the Laplace transform of the relaxation function

$$
\phi_{A B}(z)=\frac{\chi_{A B}(z)-\chi_{A B}^{T}}{i z}=\chi_{A C}^{T}\left[\frac{1}{M(z)-i z \chi^{T}}\right]_{C D} \chi_{D B}^{T},
$$

in terms of a matrix $\chi_{A B}^{T}$ of static thermodynamic susceptibilities of slow variables $A, B,{ }^{1}$ and an associated frequencydependent memory matrix $M_{A B}(z)$ (we assume that the operators $A$ and $B$ have the same sign under time reversal, otherwise an additional contribution appears in the denominator). Provided that this matrix has a finite limit $M(0)$ at vanishing frequency, this leads to an expansion

$$
\chi_{A B}(z)=\chi_{A B}^{T}+i z \chi_{A C}^{T} \tau_{C B}+\cdots
$$

of the dynamical response function at low frequencies, which defines a matrix of relaxation times

$$
\tau_{C B}=M^{-1}(0)_{C D} \chi_{D B}^{T} .
$$

Identifying $\kappa_{A B} T=\phi_{A B}(z=0)$ as the dc-transport coefficient, this leads to $\kappa_{A B} T=\chi_{A C}^{T} \tau_{C B}$, which is precisely of the form given in Eq. (1). In principle, therefore, the memory function formalism determines transport coefficients in quantum many-body systems in terms of the matrix $\chi_{A B}^{T}$ of associated static thermodynamic susceptibilities and the zero-frequency limit $M_{A B}(z=0)$ of the memory matrix. The formal expression for $M_{A B}(z)$ shows that it is again a relaxation function but now for operators $\hat{Q} \dot{A}$ in which the dynamics of the slow variables $A, B$ is projected out by $\hat{Q}=$

\footnotetext{
${ }^{1}$ Note that for nonergodic variables, the thermodynamic susceptibility $\chi_{A B}^{T}$ differs from the corresponding static one $\chi_{A B}(\omega=0)$. This results in relaxation functions that approach a nonzero limit as $t \rightarrow \infty$, a problem which does not show up for thermal transport in the unitary Fermi gas.
} 
$\left.1-\sum_{A B}\left(\chi^{T}\right)_{A B}^{-1} \mid A\right)(B \mid$. In practice, the memory matrix can hardly be determined exactly. In systems without long-lived quasiparticles, however, even approximate results for the scattering times are often sufficient because the only exactly or approximately conserved quantities are then particle number, momentum, and energy while all other variables relax on microscopic timescales.

In fact, much of the nontrivial structure of transport coefficients near quantum critical points is determined by the associated thermodynamic susceptibilities, which is behind the success of the memory function formalism in this context. This turns out to be the case also for the unitary Fermi gas studied here. Indeed, as will be shown in Sec. III, the relevant susceptibility $\chi_{q q}^{T}$ exhibits a rather strong dependence on the scaling variable $\beta \mu$ (see Fig. 4 below), while the scattering time in Fig. 5 evolves rather smoothly from the high-temperature limit down into the quantum critical regime, essentially extrapolating the result obtained from a Boltzmann equation calculation. A similar situation also applies to other transport coefficients, such as the shear viscosity $\eta=p \tau_{\eta}$, where the sum rule is given by the pressure $p$ [16], or particle transport, where an analog of the product form (1) for the thermal conductivity has also been found to hold.

In the following, we will determine the thermodynamic susceptibility $\chi_{q q}^{T}$ by a direct Green function approach, using an extension of exact Ward identities first derived by Polyakov [45] in the context of transport in the vicinity of a thermal critical point. Since the heat current is an ergodic variable, the result must coincide with the associated dc susceptibility, which is given by the standard Kramers-Kronig relation

$$
\chi_{q q}^{T}=\int \frac{d \omega}{\pi} \kappa(\omega, \mathbf{q}=0)_{\mathrm{reg}} T,
$$

as the integral of the frequency-dependent heat conductivity $\kappa(\omega, \mathbf{q}=0)$ times the temperature, including a proper regularization of the divergences which arise as a result of the assumption of a zero-range interaction in Eq. (10) below.

\section{THERMAL SUM RULE}

In this section we first introduce the model for the interacting Fermi gas in Sec. III A, and we then express the linear response theory for thermal transport in the field-theoretical formulation based on Green's functions (Sec. III B). In particular, we derive a new Ward identity for the interaction part of the heat current, which gives rise to a novel exact expression for the thermal sum rule (25)-(27) in terms of one- and twoparticle Green's functions. Next, we discuss in Sec. III C the necessary regularization of the high-momentum asymptotics. Finally, we numerically evaluate the sum rule in the quantum critical regime using the nonperturbative Luttinger-Ward approach in Sec. III D.

\section{A. Model}

The many-body physics of an ultracold Fermi gas with two trapped hyperfine states (labeled $\uparrow, \downarrow$ ) can be described by the grand canonical Hamiltonian for spin- $\frac{1}{2}$ fermions with contact interaction [10]

$$
\begin{aligned}
\hat{H}= & \int_{\mathbf{x}}\left[\sum_{\sigma=\uparrow, \downarrow} \hat{\psi}_{\sigma}^{\dagger}(\mathbf{x})\left(-\frac{\hbar^{2}}{2 m} \nabla^{2}-\mu_{\sigma}\right) \hat{\psi}_{\sigma}(\mathbf{x})\right. \\
& \left.+\bar{g}(\Lambda) \hat{\psi}_{\uparrow}^{\dagger}(\mathbf{x}) \hat{\psi}_{\downarrow}^{\dagger}(\mathbf{x}) \hat{\psi}_{\downarrow}(\mathbf{x}) \hat{\psi}_{\uparrow}(\mathbf{x})\right] .
\end{aligned}
$$

The total density is controlled by the chemical potentials, with $\mu_{\uparrow}=\mu=\mu_{\downarrow}$ in the balanced case considered here. In order to reproduce a finite $s$-wave scattering amplitude $f(k)=-1 /\left(a^{-1}+i k\right)$, the strength of the effective contact potential has to be chosen appropriately. In three dimensions, this is achieved in a standard manner by introducing a scaledependent coupling constant $\bar{g}(\Lambda)$, which is related to the physical $s$-wave scattering length $a$ via

$$
\bar{g}(\Lambda)^{-1}=m /\left(4 \pi \hbar^{2} a\right)-m \Lambda /\left(2 \pi^{2} \hbar^{2}\right) .
$$

Here, $\Lambda$ is a high-momentum cutoff whose physical origin is the finite effective range $r_{e}$ of the actual interaction. In the experimentally relevant case of open-channel dominated Feshbach resonances (e.g., for ${ }^{6} \mathrm{Li}$ at a magnetic field of $832 \mathrm{G}$ [46]) the zero-range limit $\Lambda \rightarrow \infty$ is an excellent approximation because $r_{e} \simeq \ell_{\mathrm{vdW}}$ is of the order of the van der Waals length $\ell_{\mathrm{vdW}}$ and the associated momentum scale $\Lambda \simeq 1 / \ell_{\mathrm{vdW}}$ is far beyond the accessible range [10]. As a result, the momentum distribution $n_{\sigma}(p \rightarrow \infty)=\mathcal{C} / p^{4}$ exhibits a power-law behavior over a wide range of momenta with a strength determined by the Tan contact density $\mathcal{C}$ [47]. As will be discussed below, similar power laws also appear in dynamical correlation functions. The unitary limit $1 / a=$ 0 can be reached by tuning the interaction directly to the Feshbach resonance, which is controlled by an external magnetic field. As a result, there is no small interaction parameter available and a nonperturbative treatment is mandatory to obtain quantitative results. The Luttinger-Ward approach results in single-particle Green's functions $G_{\sigma}$ at finite temperature with self-consistently resummed interaction effects and is in good agreement with thermodynamic measurements in the strong-coupling regime around the unitary limit $[37,48]$.

In addition to the fermionic Green's function $G_{\sigma}(\mathbf{x}, \tau)=$ $-\left\langle\mathcal{T}_{\tau} \hat{\psi}_{\sigma}(\mathbf{x}, \tau) \hat{\psi}_{\sigma}^{\dagger}(\mathbf{0}, 0)\right\rangle$, the Luttinger-Ward theory also allows to determine the pair propagator

$$
\begin{aligned}
\Gamma(\mathbf{x}, \tau)= & \bar{g}(\Lambda) \delta(\tau) \delta(\mathbf{x}) \\
& -\bar{g}(\Lambda)^{2}\left\langle\mathcal{T}_{\tau}\left(\hat{\psi}_{\uparrow} \hat{\psi}_{\downarrow}\right)(\mathbf{x}, \tau)\left(\hat{\psi}_{\downarrow}^{\dagger} \hat{\psi}_{\uparrow}^{\dagger}\right)(\mathbf{0}, 0)\right\rangle,
\end{aligned}
$$

where $\mathcal{T}_{\tau}$ denotes time ordering in imaginary time $\tau$. At the superfluid transition temperature $T_{c}$, the pair propagator $\Gamma\left(\mathbf{Q}=0, \Omega_{n}=0\right)$ diverges according to the Thouless criterion. Furthermore, the Tan contact is obtained from the shortdistance limit [49]

$$
\frac{\hbar^{2} \mathcal{C}}{m^{2}}-\Delta^{2}=-\Gamma\left(\mathbf{x}=0, \tau \rightarrow 0^{-}\right),
$$

where the anomalous contribution from the superfluid order parameter $\Delta$ vanishes in the normal phase considered here. In the following, both $G_{\sigma}$ and $\Gamma$ form the basis for the evaluation of the thermal sum rule. 


\section{B. Linear response}

In order to determine the thermal conductivity of the UFG, we first evaluate the thermal sum rule $\chi_{q q}^{T}$. In contrast to other transport coefficients such as the viscosity [16] or the spin diffusivity [20], $\chi_{q q}^{T}$ cannot be directly attributed to standard thermodynamic quantities but requires an additional thermal operator [50].

In general, the heat current response $\chi_{q q}(\omega)$ is obtained within linear response by adding the perturbation $\delta \hat{H}(t)=$ $\int_{\mathbf{x}} \hat{\boldsymbol{J}}^{q}(\mathbf{x}, t) \cdot \mathbf{h}(\mathbf{x}, t)$ to the Hamiltonian. Rather than working in real time, the problem is more conveniently treated in imaginary time $\tau \in[0, \hbar \beta)$. Furthermore, we consider only homogeneous source terms $\mathbf{h}(\mathbf{x}, \tau)=\mathbf{h}(\tau)$ since we are interested in the $\mathbf{q}=0$ response. We express the grand canonical partition function in the presence of the external field $\mathbf{h}$ as a coherent state path integral [51] with fermionic action $S_{F}$ :

$$
\begin{aligned}
\mathcal{Z}[\mathbf{h}]= & \int \prod_{\sigma} \mathcal{D} \bar{\psi}_{\sigma} \mathcal{D} \psi_{\sigma} e^{-\frac{1}{\hbar} S_{F}\left[\bar{\psi}_{\sigma}, \psi_{\sigma}, \mathbf{h}\right]}, \\
S_{F}\left[\bar{\psi}_{\sigma}, \psi_{\sigma}, \mathbf{h}\right]= & \int_{0}^{\hbar \beta} d \tau \sum_{\sigma} \int_{\mathbf{x}}\left(\bar{\psi}_{\sigma} \partial_{\tau} \psi_{\sigma}\right) \\
& +\int_{0}^{\hbar \beta} d \tau\left(H\left[\bar{\psi}_{\sigma}, \psi_{\sigma}\right]\right. \\
& \left.+\boldsymbol{j}^{q}(\mathbf{q}=0, \tau) \cdot \mathbf{h}(\tau)\right) .
\end{aligned}
$$

Then, $\log \mathcal{Z}$ is a generating functional for connected heat current correlations

$$
\begin{gathered}
\left\langle\hat{\boldsymbol{\jmath}}^{q}(\mathbf{q}=0, \tau)\right\rangle=-\left.\frac{\delta \log \mathcal{Z}[\mathbf{h}]}{\delta \mathbf{h}(\tau)}\right|_{\mathbf{h}=0}, \\
\chi_{q q}(\tau)=\left.\frac{\delta^{2} \log \mathcal{Z}[\mathbf{h}]}{\delta \mathbf{h}(\tau) \delta \mathbf{h}(0)}\right|_{\mathbf{h}=0} .
\end{gathered}
$$

From the latter function the retarded response in real frequency is obtained by Fourier transforming $\tau$ to the bosonic Matsubara frequency $\omega_{n}$ and subsequent analytic continuation $i \omega_{n} \rightarrow \omega+i 0^{+}$.

For the Hamiltonian (10), the particle and energy current operators read as [30,52]

$$
\begin{aligned}
\hat{\boldsymbol{j}}(\mathbf{x})= & -\frac{i \hbar}{2 m} \sum_{\sigma}\left[\psi_{\sigma}^{\dagger} \nabla \psi_{\sigma}(\mathbf{x})-\left(\nabla \psi_{\sigma}^{\dagger}\right) \psi_{\sigma}(\mathbf{x})\right], \\
\hat{\boldsymbol{j}}^{E}(\mathbf{x})= & \hbar^{3} \sum_{\sigma} \frac{\nabla \psi_{\sigma}^{\dagger} \Delta \psi_{\sigma}-\Delta \psi_{\sigma}^{\dagger} \nabla \psi_{\sigma}}{4 i m^{2}} \\
& +\frac{\hbar \bar{g}(\Lambda)}{i m} \sum_{\sigma \neq \tau} \psi_{\sigma}^{\dagger}\left[\psi_{\tau}^{\dagger} \stackrel{\leftrightarrow}{\nabla} \psi_{\tau}\right] \psi_{\sigma} .
\end{aligned}
$$

The bare energy current operator $\hat{\boldsymbol{j}}^{E}$ has a kinetic and an interaction contribution. Considering the corresponding operators in momentum space,

$$
\begin{aligned}
\hat{\boldsymbol{J}}(\mathbf{q}=0) & =\sum_{\mathbf{p} \sigma} \frac{\mathbf{p}}{m} c_{\mathbf{p}, \sigma}^{\dagger} c_{\mathbf{p}, \sigma}, \\
\hat{\boldsymbol{J}}^{E}(\mathbf{q}=0) & =\sum_{\mathbf{p} \sigma} \frac{\mathbf{p}}{m} \varepsilon_{p} c_{\mathbf{p} \sigma}^{\dagger} c_{\mathbf{p} \sigma}+\bar{g}(\Lambda) \sum_{\mathbf{Q p p} \mathbf{p}^{\prime}} \frac{\mathbf{Q}}{m} c_{\mathbf{Q} / 2+\mathbf{p} \uparrow}^{\dagger} c_{\mathbf{Q} / 2-\mathbf{p} \downarrow}^{\dagger} c_{\mathbf{Q} / 2+\mathbf{p}^{\prime} \downarrow} c_{\mathbf{Q} / 2-\mathbf{p}^{\prime} \uparrow}
\end{aligned}
$$

shows that the prefactor of the interaction part is only sensitive to the center-of-mass momentum $\mathbf{Q}$ of the pair of fermions participating in the interaction. Therefore, this term is most easily discussed in two-channel variables with a bosonic pair field $\Delta(\mathbf{x})=\bar{g} \psi_{\downarrow}(\mathbf{x}) \psi_{\uparrow}(\mathbf{x})$. The latter can be easily introduced by decoupling the action (14b) in the pairing channel by a HubbardStratonovich transformation. We notice that the presence of $\mathbf{h}(\tau)$ leads to the shift $\varepsilon_{p} \rightarrow \varepsilon_{p}+\left(\varepsilon_{p}-\mu_{\sigma}-T \tilde{s}\right) \mathbf{p} / m \cdot \mathbf{h}(\tau)$ of the bare fermionic dispersion relation and to the rescaling $\bar{g}(\Lambda) \rightarrow \bar{g}(\Lambda)[1+\mathbf{Q} / m \cdot \mathbf{h}(\tau)]$ in $S_{F}$. With these substitutions we obtain the path integral $\mathcal{Z}[\mathbf{h}]$ within the two-channel formulation in momentum space:

$$
\begin{aligned}
\mathcal{Z}[\mathbf{h}]= & \int \prod_{\sigma} \mathcal{D} \bar{c}_{\sigma} \mathcal{D} c_{\sigma} \mathcal{D} \bar{\Delta} \mathcal{D} \Delta e^{-\frac{1}{\hbar} S_{B F}\left[\bar{c}_{\sigma}, c_{\sigma}, \bar{\Delta}, \Delta, \mathbf{h}\right]}, \\
S_{B F}\left[\bar{c}_{\sigma}, c_{\sigma}, \bar{\Delta}, \Delta, \mathbf{h}\right]= & \int_{0}^{\hbar \beta} d \tau\left[\sum_{\mathbf{p}, \sigma} \bar{c}_{\mathbf{p}, \sigma}(\tau)\left(\partial_{\tau}+\varepsilon_{p}-\mu_{\sigma}+\left(\varepsilon_{p}-\mu_{\sigma}-T \tilde{s}\right) \frac{\mathbf{p}}{m} \cdot \mathbf{h}(\tau)\right) c_{\mathbf{p} \sigma}(\tau)\right. \\
& \left.-\frac{1}{\bar{g}(\Lambda)} \sum_{\mathbf{Q}}\left(1+\frac{\mathbf{Q}}{m} \cdot \mathbf{h}(\tau)\right)^{-1} \bar{\Delta}_{\mathbf{Q}}(\tau) \Delta_{\mathbf{Q}}(\tau)-\sum_{\mathbf{p}_{1}, \mathbf{p}_{2}}\left[\bar{\Delta}_{\mathbf{p}_{1}+\mathbf{p}_{2}}(\tau) c_{\mathbf{p}_{1} \downarrow}(\tau) c_{\mathbf{p}_{2} \uparrow}(\tau)+\text { H.c. }\right]\right] .
\end{aligned}
$$

From Eq. (15a), we thus find the expectation value of the heat current

$$
\left\langle\hat{\boldsymbol{J}}^{q}(\mathbf{q}=0, \tau)\right\rangle=\sum_{\mathbf{p} \sigma} \tilde{\mathcal{T}}_{\sigma}^{q(0)}(\mathbf{p}) G_{\sigma}\left(\mathbf{p}, \tau-\tau^{+}\right)-\sum_{\mathbf{Q}} \tilde{\mathcal{T}}_{\text {pair }}^{q(0)}(\mathbf{Q}) \Gamma\left(\mathbf{Q}, \tau-\tau^{+}\right),
$$

where we have defined the bare fermionic and bosonic heat current vertices

$$
\begin{aligned}
& \tilde{\mathcal{T}}_{\sigma}^{q(0)}(\mathbf{p})=\left(\varepsilon_{p}-\mu_{\sigma}-T \tilde{s}\right) \frac{\mathbf{p}}{m}, \\
& \tilde{\mathcal{T}}_{\text {pair }}^{q(0)}(\mathbf{Q})=\frac{1}{\bar{g}(\Lambda)} \frac{\mathbf{Q}}{m},
\end{aligned}
$$


and furthermore recovered the single-particle Green's function $G_{\sigma}$ as well as the pair propagator $\Gamma$ defined above. A vanishing perturbation $\mathbf{h}(\tau)=0$ implies $\left\langle\hat{\boldsymbol{J}}^{q}(\mathbf{q}=0, \tau)\right\rangle=0$ by rotation invariance. Next, we obtain the susceptibility by taking the secondorder functional derivative according to Eq. (15b):

$$
\chi_{q q}(\tau)=-\sum_{\mathbf{p} \sigma} \tilde{\mathcal{T}}_{\sigma}^{q(0)}(\mathbf{p}) \mathcal{T}_{\sigma}^{q}\left(\mathbf{0}, \tau, \mathbf{p}, 0,0^{+}\right)+\sum_{\mathbf{Q}} \tilde{\mathcal{T}}_{\text {pair }}^{q(0)}(\mathbf{Q}) \mathcal{T}_{\text {pair }}^{q}\left(\mathbf{0}, \tau, \mathbf{Q}, 0,0^{+}\right)-\frac{2 \delta(\tau)}{3 m \bar{g}(\Lambda)} \sum_{\mathbf{Q}} \frac{\mathbf{Q}^{2}}{m} \Gamma\left(\mathbf{Q}, 0,0^{+}\right) \mathbb{1}_{3 \times 3} .
$$

Here, the dressed current vertices for spin component $\sigma$ and the pairs are defined as the time-ordered expectation values

$$
\begin{gathered}
\mathcal{T}_{\sigma}^{q}\left(\mathbf{q}, \tau, \mathbf{p}, \tau_{1}, \tau_{2}\right)=\left\langle\mathcal{T}_{\tau} \hat{\boldsymbol{\jmath}}^{q}(\mathbf{q}, \tau) c_{\mathbf{p}+\mathbf{q} \sigma}\left(\tau_{1}\right) c_{\mathbf{p} \sigma}^{\dagger}\left(\tau_{2}\right)\right\rangle=\left.\frac{\delta G_{\sigma}\left(\mathbf{p}, \tau_{1}, \tau_{2}\right)}{\delta \mathbf{h}(\mathbf{q}, \tau)}\right|_{\mathbf{h}=0}, \\
\mathcal{T}_{\text {pair }}^{q}\left(\mathbf{q}, \tau, \mathbf{Q}, \tau_{1}, \tau_{2}\right)=\left\langle\mathcal{T}_{\tau} \hat{\boldsymbol{\jmath}}^{q}(\mathbf{q}, \tau) \Delta_{\mathbf{Q}+\mathbf{q}}\left(\tau_{1}\right) \Delta_{\mathbf{Q}}^{\dagger}\left(\tau_{2}\right)\right\rangle=\left.\frac{\delta \Gamma\left(\mathbf{Q}, \tau_{1}, \tau_{2}\right)}{\delta \mathbf{h}(\mathbf{q}, \tau)}\right|_{\mathbf{h}=0},
\end{gathered}
$$

while the last contribution to $\chi_{q q}$ arises from the second derivative of the $\bar{\Delta} \Delta$ prefactor in the action (18b). The thermal sum rule follows by Fourier transformation to the external bosonic Matsubara frequencies $\omega_{n}=0$, which yields

$$
\begin{aligned}
\chi_{q q}\left(\omega_{n}=0\right)= & -\sum_{\mathbf{p} \sigma, \epsilon_{m}} \tilde{\mathcal{T}}_{\sigma}^{q(0)}(\mathbf{p}) \mathcal{T}_{\sigma}^{q}\left(\mathbf{0}, \omega_{n}=0, \mathbf{p}, \epsilon_{m}\right)+\sum_{\mathbf{Q}, \Omega_{m}} \tilde{\mathcal{T}}_{\text {pair }}^{q(0)}(\mathbf{Q}) \mathcal{T}_{\text {pair }}^{q}\left(\mathbf{0}, \omega_{n}=0, \mathbf{Q}, \Omega_{m}\right) \\
& -\frac{2}{3 m \bar{g}(\Lambda)} \sum_{\mathbf{Q}, \Omega_{m}} \frac{\mathbf{Q}^{2}}{m} \Gamma\left(\mathbf{Q}, \Omega_{m}\right) \mathbb{1}_{3 \times 3} .
\end{aligned}
$$

This form of the sum rule in terms of current vertex functions is analogous to the sum rules for momentum [16,53,54] and spin currents [55]. Introducing $\tilde{\mathcal{T}}_{\sigma \text {,pair }}^{q}$ as the amputated counterparts of $\mathcal{T}_{\sigma \text {,pair }}^{q}$ allows one to represent the Kubo formula for $\chi_{q q}$ and thus also the sum rule in a diagrammatic manner, as depicted in Fig. 3, except for the last line.

Quite crucially, the exact heat current vertex $\tilde{\mathcal{T}}^{q}$ satisfies a Ward identity [45]. Extending the latter from the fermionic to the bosonic sector, it reads in momentum space (cf. Appendix A) as

$$
\begin{gathered}
\tilde{\mathcal{T}}_{\sigma}^{q}(\mathbf{p}, \varepsilon)=(T \tilde{s}-\varepsilon) \frac{\partial G_{\sigma}^{-1}(\mathbf{p}, \varepsilon)}{\partial \mathbf{p}}-\frac{\mathbf{p}}{m} G_{\sigma}^{-1}(\mathbf{p}, \varepsilon), \\
\tilde{\mathcal{T}}_{\text {pair }}^{q}(\mathbf{Q}, \Omega)=-\Omega \frac{\partial \Gamma^{-1}(\mathbf{Q}, \Omega)}{\partial \mathbf{Q}}+\frac{\mathbf{Q}}{m} \Gamma^{-1}(\mathbf{Q}, \Omega),
\end{gathered}
$$

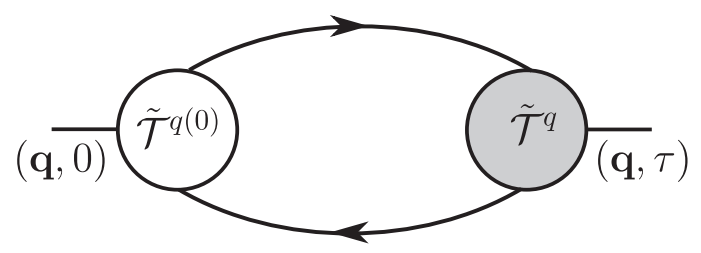

$$
\tilde{\mathcal{T}}^{q}\left(\mathbf{q}, \tau, \mathbf{p}, \tau_{1}, \tau_{2}\right)=\overbrace{(\mathbf{q}, \tau)}^{\left(\mathbf{p}+\mathbf{p}, \tau_{1}\right)}
$$

FIG. 3. Diagrammatic representation of the current correlation function (Kubo formula) and the dressed, amputated current vertex $\tilde{\mathcal{T}}^{q}$. The total response is given by the sum of the fermionic and pair contributions. at vanishing external arguments $\omega=0, \mathbf{q} \rightarrow \mathbf{0}$ relevant for the sum rule. The first line contains the fermionic part expressed via the single-particle Green's function $G_{\sigma}$, while the second line denotes the bosonic contribution in terms of the pair propagator $\Gamma$. The bare vertices $\tilde{\mathcal{T}}_{\sigma}^{q(0)}$ and $\tilde{\mathcal{T}}_{\text {pair }}^{q(0)}$ in Eq. (20) are obtained simply by using the noninteracting Green's function $G_{0, \sigma}^{-1}(p, \varepsilon)=\varepsilon-\varepsilon_{p}+\mu$ and the inverse bare coupling $\Gamma_{0}^{-1}(Q, \Omega)=\bar{g}(\Lambda)^{-1}$ inside the Ward identity (see Appendix A).

In order to obtain the sum rule as the static limit of the current response function given by the Kubo formula (Fig. 3) we insert the Ward identities into Eq. (23). As a result, we obtain the exact thermal sum rule expressed in terms of the Green's and vertex functions

$$
\bar{\chi}_{q q}^{T}(T, \mu)=\bar{\chi}_{q q}^{T, \mathrm{~F}}(T, \mu)+\bar{\chi}_{q q}^{T, p a i r}(T, \mu),
$$

with two contributions: a fermionic part

$$
\begin{aligned}
\bar{\chi}_{q q}^{T, \mathrm{~F}}= & -\frac{1}{\beta V} \sum_{p \sigma \epsilon_{n}} \frac{p^{2}}{3 m^{2}}\left(\varepsilon_{p}-\mu-T \tilde{s}\right) \\
& \times\left[\left(i \epsilon_{n}-T \tilde{s}\right) \frac{m}{p} \frac{\partial G_{\sigma}\left(p, i \epsilon_{n}\right)}{\partial p}-G_{\sigma}\left(p, i \epsilon_{n}\right)\right]
\end{aligned}
$$

and a new interaction part arising from the bosonic pairs of the form

$$
\begin{aligned}
\bar{\chi}_{q q}^{T, \text { pair }}= & \left(\frac{m}{4 \pi \hbar^{2} a}-\frac{m \Lambda}{2 \pi^{2} \hbar^{2}}\right) \\
& \times \frac{1}{\beta V} \sum_{Q \Omega_{n}} \frac{1}{m}\left(\frac{Q^{2}}{3 m}-i \Omega_{n}\right) \Gamma\left(Q, i \Omega_{n}\right) .
\end{aligned}
$$

Both terms can be evaluated by inserting the previously computed Luttinger-Ward results for $G_{\sigma}\left(p, i \varepsilon_{n}\right)$ and $\Gamma\left(Q, i \Omega_{n}\right)$ [32-34] as functions of momentum $p(Q)$ and Matsubara frequency $i \varepsilon_{n}\left(i \Omega_{n}\right)$. 
Note that the full fermionic and bosonic energy current vertices as defined by the Ward identity (24) provide an exact solution of the Luttinger-Ward transport equations formulated in terms of fermionic and bosonic transport vertices [16,54]. This proves that the Luttinger-Ward approach implements exact energy conservation, even when fermionic and bosonic Green's functions are obtained within the self-consistent $T$ matrix approximation. This was indeed the goal of constructing a conserving approximation, which in our case furthermore satisfies the exact Tan relations [36].

However, as indicated by the bar, these terms still depend explicitly on the momentum cutoff $\Lambda$, which is manifest for the second term. Moreover, a finite value of $\Lambda$ is necessary to render the momentum integrals in the fermionic part finite. Therefore, we first have to discuss how to extract the universal results for the sum rule before presenting the numerical results.

\section{Short-distance asymptotics}

Due to the contact interaction, several terms in the sum rule (26) and (27) diverge in the zero-range limit $\Lambda \rightarrow \infty$. This is apparent for the pair contribution, which in the unitary limit $1 / a=0$ is directly proportional to $\Lambda$. Indeed, one quite generally expects a cutoff dependence of the static sum rules for these quantum critical systems. This can be attributed to high-frequency tails of the dynamic transport coefficients such as $\kappa(\omega)$ defined in Eq. (5) above [6]. For instance, in case of the shear viscosity the full sum rule reads as $[16,53]$

$$
\left\langle\hat{\Pi}_{x y} \hat{\Pi}_{x y}\right\rangle_{\omega=0}=p+\frac{4 \hbar^{2} \mathcal{C} \Lambda}{15 \pi^{2} m},
$$

where the second term arises from the high-frequency tail $\eta(\omega \rightarrow \infty)=\hbar^{3 / 2} \mathcal{C} / 15 \pi \sqrt{m \omega}$. The static transport coefficient $p$ is given instead by the regularized form of the sum rule with the $\Lambda$-dependent terms subtracted. As a result for the shear viscosity of the unitary gas, one has $\eta=p \tau_{\eta}$ in analogy to Eq. (1) in the thermal case.

These divergences arise from the asymptotic largemomentum behavior of the fermionic momentum distribution

$$
n_{\sigma}(p \rightarrow \infty) \sim \mathcal{C} / p^{4}+\mathcal{D}_{1} / p^{6}+\mathcal{D}_{2} / p^{7}+\cdots,
$$

where we identify a new contribution $\mathcal{D}_{2} / p^{7}$ that is clearly seen in our numerical data. As discussed in Appendix B, its origin may be traced back to a next-to-leading-order nonanalytical contribution $\Gamma\left(\mathbf{x}=\mathbf{0}, \tau \rightarrow \beta^{-}\right) \sim(\beta-\tau)^{3 / 2}$ in the pair propagator at short times. The appearance of the two leading contributions in the asymptotic power-law decay of the momentum distribution arises from the nonanalytic contributions proportional to $|\mathbf{x}|$ and $|\mathbf{x}|^{3}$, respectively, in the short-distance operator product expansion

$$
\begin{aligned}
\hat{\psi}_{\sigma}^{\dagger}\left(\mathbf{R}+\frac{\mathbf{x}}{2}\right) \hat{\psi}_{\sigma}\left(\mathbf{R}-\frac{\mathbf{x}}{2}\right) \\
=\hat{n}_{\sigma}(\mathbf{R})+i \hbar^{-1} \mathbf{x} \cdot \hat{\mathbf{p}}_{\sigma}(\mathbf{R}) \\
\quad-\frac{|\mathbf{x}|}{8 \pi} \hbar^{-4} m^{2} \bar{g}^{2}(\Lambda) \hat{\psi}_{\uparrow}^{\dagger} \hat{\psi}_{\downarrow}^{\dagger} \hat{\psi}_{\downarrow} \hat{\psi}_{\uparrow}(\mathbf{R}) \\
\quad+\frac{|\mathbf{x}|^{3}}{96 \pi} \hbar^{-4} m^{2} \bar{g}^{2}(\Lambda) \nabla_{\mathbf{R}}^{2} \hat{\psi}_{\uparrow}^{\dagger} \hat{\psi}_{\downarrow}^{\dagger} \hat{\psi}_{\downarrow} \hat{\psi}_{\uparrow}(\mathbf{R})+\cdots
\end{aligned}
$$

of the one-particle density matrix [56]. The coefficients $\mathcal{C}$ and $\mathcal{D}_{1}$ in Eq. (29) are defined through the expectation values of the contact operator $\hat{\mathcal{C}}(\mathbf{R})=\hbar^{-4} m^{2} \bar{g}^{2}(\Lambda) \hat{\psi}_{\uparrow}^{\dagger} \hat{\psi}_{\downarrow}^{\dagger} \hat{\psi}_{\downarrow} \hat{\psi}_{\uparrow}(\mathbf{R})$ and its second derivative $\nabla_{\mathbf{R}}^{2} \hat{\mathcal{C}}(\mathbf{R})$; note that in $d=3$ the Fourier transform of $|\mathbf{x}|$ is $-8 \pi / p^{4}$ while $|\mathbf{x}|^{3}$ gives $96 \pi / p^{6}$. The presence of a subleading term $\mathcal{D}_{1} / p^{6}$ in the momentum distribution of two-component Fermi gases has been discussed in detail by Werner and Castin [57]. In general, the coefficient $\mathcal{D}_{1}$ also contains a contribution which involves the derivative of the energy with respect to the effective range of the interaction. In our model, no such contribution appears and the full expression for $\mathcal{D}_{1}$ is given in terms of the first-order time and second-order spatial derivative of the pair propagator [see Eqs. (B5) and (B7) in Appendix B].

Within the self-consistent $T$-matrix approximation to the Luttinger-Ward functional the powers of momentum are correctly reproduced, whereas the contact coefficients $\mathcal{C}, \mathcal{D}_{1}$, and $\mathcal{D}_{2}$ that characterize the short-distance correlations as functions of $T, \mu$, and $a^{-1}$ in the many-body medium are obtained approximately. The asymptotic behavior of the numerical data of the fermionic momentum distribution is consistent both with the OPE and Ref. [57] up to $p^{-6}$, but to our knowledge the $p^{-7}$ contribution has not been discussed before. The latter arises from an anomalous contribution to the pair propagator $\Gamma\left(\mathbf{x}=\mathbf{0}, \tau \rightarrow \beta^{-}\right) \sim(\beta-\tau)^{3 / 2}$ (see Appendix B).

In the fermionic part (26), the leading divergence $O\left(\Lambda^{3}\right)$, which could arise from the $\mathcal{C} / p^{4}$ tail of the momentum distribution, cancels between the first and last term in the square brackets, hence, there is no $\Lambda^{3}$ divergence. According to Eq. (29), this leaves terms of order $O(\Lambda)$ and $O(\log (\Lambda / \bar{k}))$, where $\bar{k}$ denotes the momentum scale beyond which the algebraic power laws of the terms in $\chi_{q q}^{T}$ dominate; in practice, one has $\bar{k} \gtrsim 10 / \lambda_{T}$. The coefficients of these subleading divergences depend on $\mathcal{C}, \mathcal{D}_{1}$, and $\mathcal{D}_{2}$ (for the $\log$ term). Similarly, for the pair momentum distribution we find the asymptotic expansion $n_{\text {pair }}(Q \rightarrow$ $\infty)=64 \pi^{2} n \mathcal{C} / 3 Q^{6}+\cdots$ [see Eq. (B12) in Appendix B]. This implies that the momentum sum in the interaction term (27) is finite, while the inverse bare coupling in the prefactor diverges as $O(\Lambda)$. In the numerical evaluation, we subtract all divergent terms to obtain the regularized sum rule (9), as has been done for the shear viscosity [16]. At unitary, in particular, the interaction term does not contribute to the regularized sum rule as its contribution scales like $1 / a$. Away from unitarity $1 / a \neq 0$, in turn, the bosonic part gives rise to a new contact correlation contribution to the thermal conductivity similar to what has been found in the bulk viscosity $[54,58,59]$. Regarding the dynamic thermal conductivity, the $O(\Lambda)$ contribution implies a tail $\kappa(\omega \rightarrow \infty) \sim \omega^{-1 / 2}$, while $O(\ln \Lambda)$ causes a subleading contribution to the high-frequency behavior proportional to $\omega^{-1}$, in analogy to the discussion below Eq. (28).

\section{Numerical results}

After subtracting from Eq. (25) all terms that diverge in the zero-range limit, we find the exact result for the thermal conductivity sum rule at unitarity where the pair contribution 


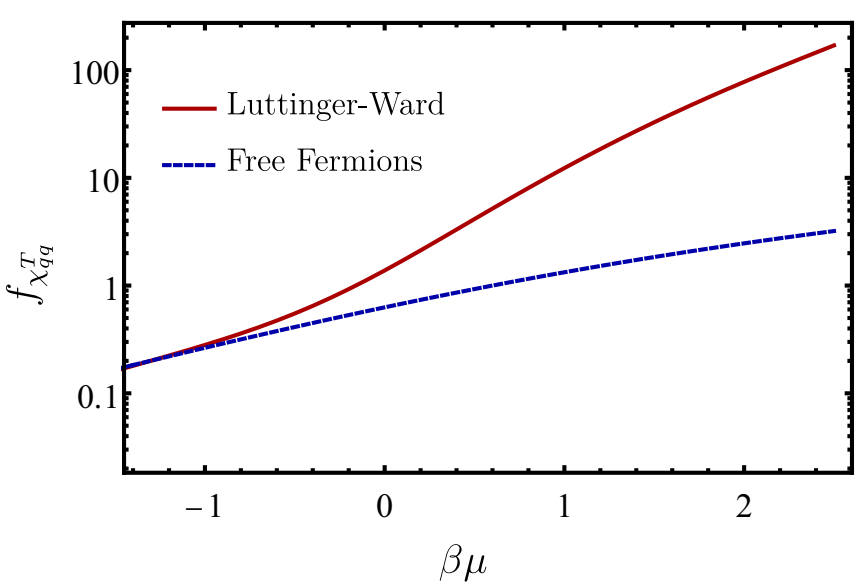

FIG. 4. Thermal sum rule scaling function $f_{\chi_{q q}^{T}}$ vs $\beta \mu$ for the unitary Fermi gas: the interacting Luttinger-Ward result (25) is substantially larger than the free-fermion result (32) in the quantum degenerate regime.

(27) vanishes:

$$
\chi_{q q}^{T}(T, \mu)=\chi_{q q}^{T, \mathrm{~F}}(T, \mu) \equiv \frac{T^{3}}{\hbar^{2} \lambda_{T}} f_{\chi_{q q}^{T}}(\beta \mu) .
$$

This defines the dimensionless quantum critical scaling function $f_{\chi_{q q}^{T}}(\beta \mu)$. In the high-temperature regime the sum rule is given analytically by the result

$$
\begin{aligned}
\chi_{q q}^{T(0)}(T, \mu)= & -\frac{1}{4 \pi} \frac{T^{3}}{\hbar^{2} \lambda_{T}}\left[35 \operatorname{Li}_{7 / 2}\left(-e^{\beta \mu}\right)\right. \\
& \left.-10\left(\beta \mu+\tilde{s}^{(0)}\right) \operatorname{Li}_{5 / 2}\left(-e^{\beta \mu}\right)\right]
\end{aligned}
$$

of a free Fermi gas. The entropy per particle is $\tilde{s}_{(0)}=$ $5 \mathrm{Li}_{5 / 2}\left(-e^{\beta \mu}\right) /\left[2 \mathrm{Li}_{3 / 2}\left(-e^{\beta \mu}\right)\right]-\beta \mu$, where $\operatorname{Li}_{s}(z)$ denotes the polylogarithm. In terms of density, the result approaches $m \chi_{q q}^{T} \rightarrow(5 / 2) n T^{2}$ for $\beta \mu \rightarrow-\infty$. Using the Luttinger-Ward thermodynamic data allows us to extend the sum rule from the high-temperature regime into the quantum degenerate regime down to the critical temperature of the superfluid transition at $(\beta \mu) \simeq 2.5$ [37]. To obtain reliable results after the subtraction of the nonintegrable, cutoff-dependent tails requires a precise calculation of the self-consistent Green's and vertex functions. This has been accomplished by using a logarithmic Fourier transform $[60,61]$ for the transformation between real and momentum space, while the imaginary time to Matsubara frequency transformation is performed by a discrete Fourier transform in combination with a spline interpolation up to fifth order [34]. Consistency checks on thermodynamic variables such as pressure, which contain integrals over the momentum tails as in Eq. (26) that must remain finite in the zero-range limit $\Lambda \rightarrow \infty$, show relative numerical errors of at most $10^{-3}$ in the regime considered here. This level of accuracy is a crucial prerequisite for dealing with the more complicated asymptotics encountered in the thermal sum rule by subtraction of the known high-momentum behavior of the Green and vertex functions, as discussed in detail in Appendix B. The result for $\chi_{q q}^{T}$ is shown in Fig. 4: while it agrees with $\chi_{q q}^{T(0)}$ in the virial limit, it shows large deviations in the quantum degenerate regime from strong pairing fluctuations, which lead to an enhancement of up to two orders of magnitude close to the superfluid transition.

\section{QUANTUM CRITICAL SCATTERING TIMES}

Within kinetic theory, the thermal scattering time $\tau_{\kappa}$ is obtained as the collision time in the Boltzmann equation in response to temperature gradients $[62,63]$. While this approach becomes exact in the virial limit of a nondegenerate, extremely dilute gas, it can be formally justified in the QCR only within a large- $N$ expansion in the number of fermion flavors, thereby restoring a quasiparticle picture [36]. Quite surprisingly, however, this method not only yields a qualitative estimate on the physical behavior of the scattering times in the QCR, it also allows to find a simple but quantitative approximation as we argue below. In the Boltzmann equation the collision integral $I[f]$ is evaluated for a generic distribution function $f_{p}$, which deviates from the thermal equilibrium distribution $f_{p}^{0}$ as $f_{p}=f_{p}^{0}+\delta f_{p}$. For small variations $\delta f_{p}$, the collision integral can be linearized as $I\left[f_{p}\right] \approx H\left[f_{p}^{0}\right] \delta f_{p}$, where the linearized collision operator $H\left[f_{p}^{0}\right]$ acts on $\delta f_{p}$ but itself only depends on the equilibrium distribution $f_{p}^{0}$. The solution $\delta f_{p}=f_{p}^{0}\left(1-f_{p}^{0}\right) U_{p}$ of the Boltzmann equation minimizes the scattering rate, hence, the particles choose a distribution $U_{p}$ to best avoid scattering. Within a family of trial functions $U_{p}$, an upper bound to the true scattering rate is found in variational kinetic theory as $[62,64]$ (for further details see also Appendix C)

$$
\tau^{-1}=\min _{U_{p}} \frac{(U, H U)(X, X)}{(U, X)^{2}} .
$$

The scalar products $(A, B)=\int d \Gamma_{p} f_{p}^{0}\left(1-f_{p}^{0}\right) A_{p} B_{p}$ are defined with respect to the equilibrium distribution function $f_{p}^{0}$. The system is driven out of equilibrium by the perturbation $X_{p}$ : it determines which transport channel is considered, e.g., $X_{p}=\frac{p_{z}}{m}\left[\varepsilon_{p}-(T \tilde{s}+\mu)\right]$ for thermal and $X_{p}=\frac{p_{x} p_{y}}{m}$ for shear transport. The variational functions $U_{p}$ are arbitrary functions of momentum that have the same angular dependence as the perturbation $X_{p}$.

The linear collision operator $H\left[f^{0}\right]$ for $2 \rightarrow 2$ scattering between fermionic (quasi)particles $\left(\boldsymbol{p}_{1}, \boldsymbol{p}_{2} \mapsto \boldsymbol{p}_{1^{\prime}}, \boldsymbol{p}_{2^{\prime}}\right)$ reads as

$$
H\left[f_{1}^{0}\right]=\int d \Gamma_{2} d \Omega \frac{d \sigma\left[f^{0}\right]}{d \Omega}\left|\boldsymbol{v}_{1}-\boldsymbol{v}_{2}\right| f_{2}^{0}\left(1-f_{1^{\prime}}^{0}\right)\left(1-f_{2^{\prime}}^{0}\right),
$$

where momentum conservation $\boldsymbol{p}_{1}+\boldsymbol{p}_{2}=\boldsymbol{p}_{1^{\prime}}+\boldsymbol{p}_{2^{\prime}}$ and energy conservation $\varepsilon_{p_{1}}+\varepsilon_{p_{2}}=\varepsilon_{p_{1^{\prime}}}+\varepsilon_{p_{2^{\prime}}}$ are satisfied in elastic scattering, and $\Omega$ denotes the angle between the incoming and outgoing scattering planes. The scattering cross section is given as $d \sigma / d \Omega=|\tilde{f}|^{2}$ in terms of the $s$-wave scattering amplitude $\tilde{f}$; in the strongly interacting Fermi gas, the medium scattering amplitude reads as [34,36]

$$
\begin{aligned}
-\tilde{f}^{-1}= & \frac{1}{a}+i \frac{\left|\boldsymbol{p}_{1}-\boldsymbol{p}_{2}\right|}{2} \\
& +\int d \Gamma_{p} \frac{2 f_{p}^{0}}{\varepsilon_{p_{1}}+\varepsilon_{p_{2}}-\varepsilon_{p}-\varepsilon_{\boldsymbol{p}_{1}+\boldsymbol{p}_{2}-\boldsymbol{p}}+i 0}
\end{aligned}
$$




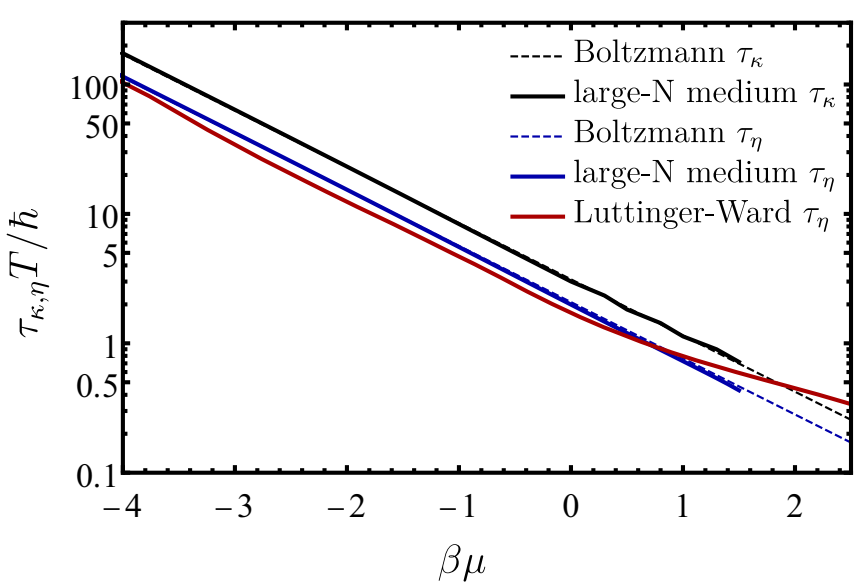

FIG. 5. Thermal and viscous scattering times $\tau_{\kappa, \eta} T / \hbar$ vs $\beta \mu$ in the quantum critical region of the unitary Fermi gas. In this regime, both Boltzmann and large- $N$ calculations give nearly identical results. Furthermore, the large- $N$ viscous scattering time (blue) agrees well even with the strong-coupling Luttinger-Ward computation [16] (red).

to leading order in the systematic large- $N$ expansion. While the first two terms reproduce the $s$-wave scattering amplitude at the two-particle level, the integral takes corrections caused by the presence of a finite density medium into account. At unitarity $1 / a=0$ the constant offset vanishes, and the dimensionless scattering amplitude $\tilde{f} / \lambda_{T}$ depends on $\beta \mu$ alone.

The properties at high temperature are obtained to leading order in the virial expansion in small fugacity $z=e^{\beta \mu} \ll 1$, with $f_{p}^{0}=e^{-\beta\left(\varepsilon_{p}-\mu\right)}$ the Boltzmann distribution. The resulting scattering times are

$$
\frac{\tau_{\kappa} T}{\hbar}=\frac{45 \pi}{32 \sqrt{2}} e^{-\beta \mu}
$$

and $\tau_{\eta} T / \hbar=\frac{15 \pi}{16 \sqrt{2}} e^{-\beta \mu}$ [64] already from the first variational basis function $U_{p} \propto X_{p}$, and corrections from higher basis functions are less than $1.5 \%$ for the shear viscosity [65]. Note that the high-temperature results at unitarity already satisfy the quantum critical scaling form $\tau_{x} T / \hbar=f_{x}(\beta \mu)$ with $x=\kappa, \eta$.

In the quantum degenerate regime, one instead has to use the Fermi-Dirac distribution $f_{p}^{0}=\left[e^{\beta\left(\varepsilon_{p}-\mu\right)}+1\right]^{-1}$. Two competing effects thus modify the scattering times $\tau_{x}$ : Pauli blocking in Eq. (34) reduces the phase space for scattering and strongly increases the scattering time, while medium scattering in Eq. (35) has the opposite effect and reduces the scattering time. In case of the shear viscosity, the scattering cross section $d \sigma / d \Omega$ even diverges at the superfluid transition due to gapless pairing fluctuations if only a single variational basis function $U_{p} \propto X_{p}$ is considered. This would lead to the unphysical result $\eta \rightarrow 0$ at $T_{c}$, which arises from the divergence of the $T$ matrix $\Gamma \sim Q^{-2}$ for small energies. However, an improved variational solution in a larger basis set yields a finite result, as is expected for the viscosity near the superfluid transition [40].

The full results are shown in Fig. 5: the surprising and remarkable observation is that the scattering time $f_{x}(\beta \mu)$ is nearly the same for the Boltzmann distribution ("Boltzmann") and for the Fermi-Dirac distribution ("large- $N$ medium"), not only for viscous [66] but also for thermal transport. Changing the distribution $f_{p}^{0}$ from Boltzmann to Fermi-Dirac modifies the calculation in three places: (i) in the scalar product in the variational expression (33), (ii) in the occupation numbers of the collision integral (34), and finally (iii) in the medium scattering amplitude (35). In the quantum critical regime, the subtle interplay between these effects leads to an almost perfect cancellation between the Pauli blocking and medium scattering corrections in the large- $N$ medium result. We find a similar coincidence also for spin diffusion (see Appendix C). Hence, there appears to be a general mechanism at work that does not depend on the angular, spin, or energy weight of the driving term $X_{p}$.

What has not been appreciated before is that, even more remarkably, also the strong-coupling Luttinger-Ward computations [16] (red) confirm this result for the scattering time as a function of $\beta \mu$ for the whole quantum critical regime $\beta \mu \lesssim 1$ $\left(T \gtrsim 2 T_{c}\right.$ ) within a $15 \%$ error bound, where the scattering time has been extracted from the relation $\eta=p \tau_{\eta}$ in analogy to Eq. (1). We thus conjecture that the large- $N$ expansion is similarly accurate for the thermal scattering time $\tau_{\kappa}$ in the quantum critical regime, and we use the large- $N$ result (36) henceforth. Closer to the phase transition, however, the quantum critical scaling crosses over into the classical critical scaling of the 3D XY universality class near the superfluid phase transition (see Sec. II A above).

At unitarity, the scattering times thus satisfy the quantum critical scaling form [5,36] $\tau_{x}=f_{x}(\beta \mu)(\hbar / T)$, where the dimensionless scaling function $f_{x}(\beta \mu)$ depends only on the value of $\beta \mu$, not only in the quantum critical regime but also in the high-temperature nondegenerate gas; in the quantum degenerate region $\beta \mu \geqslant 0$, the scaling function attains values of order unity (Fig. 5). For spin transport (Appendix C), this is consistent with the experimental observation of quantum critical spin drag [19] and Planckian dissipation for spin $[21,22,67]$.

\section{RESULTS AND QUANTUM CRITICAL TRANSPORT RATIOS}

Based on the hydrodynamic arguments from above, we arrive at the first prediction for the thermal conductivity (1), $\kappa T=\chi_{q q}^{T} \tau_{\kappa}$, in the quantum critical regime, as shown in Fig. 6. Here, $\chi_{q q}^{T}$ is evaluated within Luttinger-Ward theory (Fig. 4) and combined with the thermal scattering time in the Boltzmann limit (36). In the limit $\beta \mu \rightarrow-\infty$ one finds the Boltzmann value for the thermal conductivity $\kappa^{B}=$ $225 /(64 \sqrt{2}) T /\left(\hbar \lambda_{T}\right)$ [31] by using the noninteracting sum rule (32). At lower temperatures, however, the strong enhancement of the thermal sum rule implies a significantly larger thermal conductivity as opposed to the result from the Boltzmann equation (cf. Fig. 4).

Weighting the thermal diffusion $D_{T}$ with the thermodynamic Landau-Placzek ratio $\mathrm{LP}=c_{p} / c_{V}-1$ (Fig. 7) yields the thermal contribution $\mathrm{LP} \times D_{T}$ to the sound diffusion $D_{\text {sound }}$ shown in Fig. 1. At low temperatures above $T_{c}$ the decrease of LP seems to suggest that heat diffusion becomes less important for sound attenuation near $T_{c}$, but this is more 


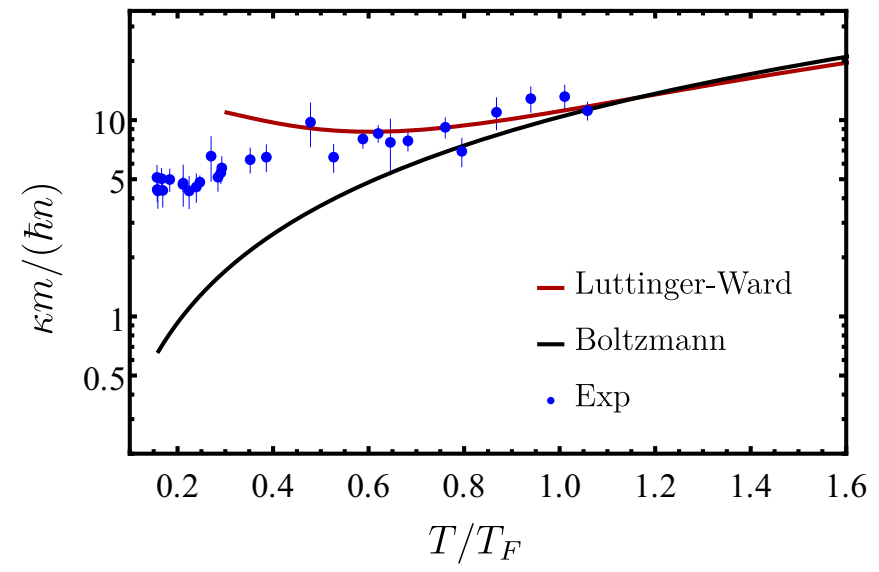

FIG. 6. Thermal conductivity $\kappa$ vs temperature $T / T_{F}$ for the unitary Fermi gas from Luttinger-Ward calculations (red line) and from experiment [23]. $\kappa$ saturates in the quantum degenerate regime and exhibits a shallow minimum of $\kappa / n \approx 8.7 \hbar / m$ at $T / T_{F} \approx 0.6$.

than compensated by the increase of $D_{T}$ which makes heat diffusion rather more important.

A fluid is characterized by the relative importance of different transport channels, which is quantified by transport ratios. Here, we consider the Prandtl number, which is defined as the ratio of shear and thermal diffusivities $\left(D_{T}\right.$ is reported in Fig. 1)

$$
\operatorname{Pr}=\frac{D_{\eta}}{D_{T}}=\frac{(p / m n) \tau_{\eta}}{\left(\chi_{q q}^{T} / c_{p} T\right) \tau_{\kappa}}=\frac{p c_{p} T}{m n \chi_{q q}^{T}} \times \frac{\tau_{\eta}}{\tau_{\kappa}} .
$$

As the last term shows, the transport ratio is a product of a thermodynamic term that incorporates nontrivial temperature scaling from the full equation of state, and a ratio of transport times which we have found to remain nearly constant at $\tau_{\eta} / \tau_{\kappa}=\frac{2}{3}$ throughout the quantum critical regime. Therefore, in the unitary gas the transport ratios derive their temperature dependence predominantly from the equation of state, and we use the best available Luttinger-Ward equation of state

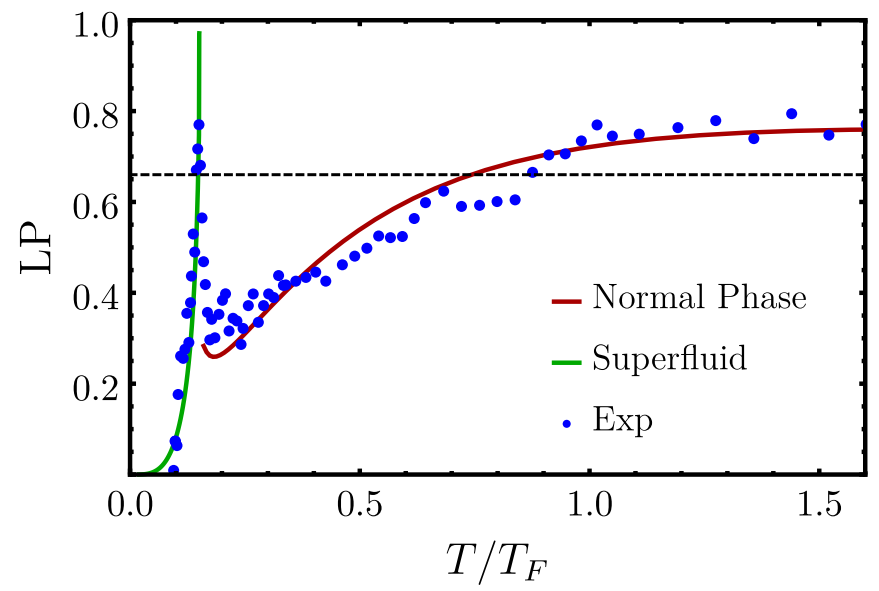

FIG. 7. Landau-Placzek ratio $\mathrm{LP}=c_{p} / c_{V}-1$ vs temperature $T / T_{F}$ from Luttinger-Ward calculations (green below $T_{c}$ [32], red above $T_{c}$ [34]) and from experiment [37]. The dashed line indicates the high-temperature limit $\mathrm{LP}=\frac{2}{3}$.

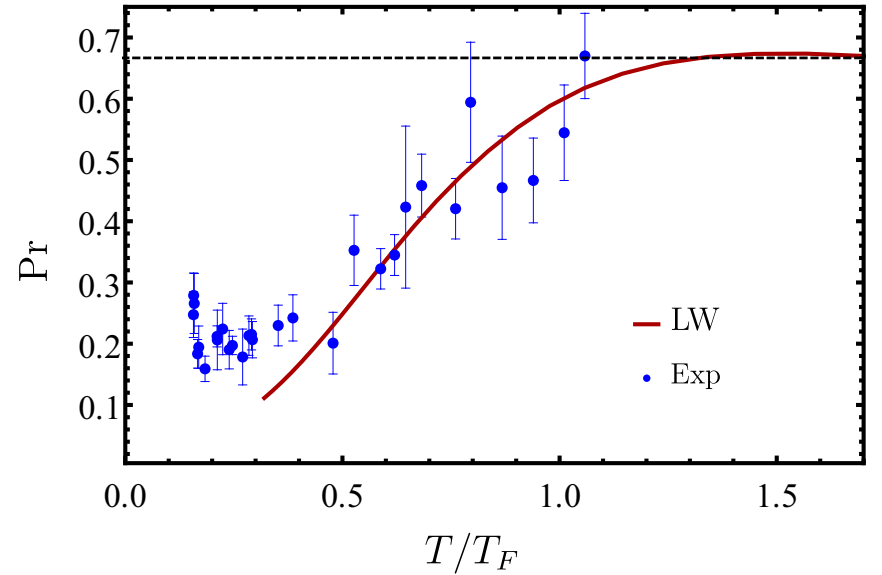

FIG. 8. Prandtl number $\operatorname{Pr}=D_{\eta} / D_{T}$ vs temperature $T / T_{F}$ from Luttinger-Ward calculations (red line) and sound attenuation measurements [23]; the dashed line marks the high-temperature limit $\operatorname{Pr}=\frac{2}{3}$.

$[32,34]$ to obtain the theory prediction for the Prandtl number in Fig. 8. Note that Pr starts from a value of $\frac{2}{3}$ in the hightemperature limit and then grows to about 0.7 near $T \approx T_{F}$, before it falls to much smaller values below 0.2 near the superfluid transition. This nonmonotonic behavior results from the Landau-Placzek ratio [26,34] shown in Fig. 7 for the unitary Fermi gas, and is consistent with the virial expansion [31]. At the classical superfluid phase transition (model F) [40] one expects that $\eta$ remains finite while $\kappa$ diverges according to Eq. (2), suggesting a vanishing $\operatorname{Pr} \rightarrow 0$. This nonmonotonic behavior is very well confirmed by a recent measurement of sound attenuation in the unitary gas [23]. The value of the Prandtl number also has an important interpretation in terms of possible nonrelativistic gravity duals, which, however, predict $\mathrm{Pr}=1$ [68] and can therefore be excluded as a model for the unitary Fermi gas. Another important transport ratio is the bulk-to-shear viscosity ratio $\zeta / \eta$ computed in [54], which shows that viscous transport occurs via quasiparticles only for $T \gtrsim T_{F}$ but deviates in the quantum degenerate regime. The Schmidt number $\mathrm{Sc}=D_{\eta} / D_{s}$ comparing shear with spin transport is shown in Fig. 10 (see Appendix C).

\section{DISCUSSION}

In conclusion, we have found that transport scattering times $\tau_{\kappa}$ and $\tau_{\eta}$ in the quantum critical regime follow a remarkably simple scaling law, which extends to the vicinity of the superfluid transition where pairing fluctuations become dominant. We have chosen specifically the unitary gas where the quantum critical regime extends to high temperature to demonstrate this point. This information is combined with a new exact sum rule for thermal transport, which depends on the equation of state and thermal operators beyond, to predict the thermal conductivity $\kappa$ in the quantum degenerate regime. For $\kappa$ and the Prandtl number Pr we find good agreement with recent experiments [23].

The remarkable coincidence of the quantum critical scattering times from the high-temperature Boltzmann calculation and the strong-coupling large- $N$ and Luttinger-Ward results 
is a unique feature of the quantum critical point at infinite scattering length $1 / a=0$ : the scattering times must follow the quantum critical scaling form, which in the particular case of the unitary Fermi gas must extend up to high temperature by dimensional analysis, in contrast to lattice models. At high temperature, the scattering times are reliably obtained from kinetic theory as $\tau T / \hbar \propto z^{-1}$ proportional to the inverse fugacity. Now, quantum critical scaling predicts that this form continues from the dilute gas throughout the QCR until near $T_{c}$. This remarkable observation is supported by the fact that it leads to good agreement with recent experimental data in the regime where quantum critical scaling can be applied. It will be interesting to see if our approach can be extended to other types of QCPs.

While at unitarity the bosonic part of the exact sum rule (25) provides only a regularization, away from unitarity $(1 / a \neq 0)$ it gives a new regular contribution that arises from local pair fluctuations, the so-called contact correlations [54]. This new contribution to thermal transport is not captured by fermionic kinetic theory and is particularly important at low temperatures near the superfluid phase transition.

We find that not only shear and spin diffusion, but also the thermal diffusion $D_{T}$ in units of $\hbar / m$ exhibit quantum limited diffusion near $T_{c}$. For thermal transport, the diffusion minimum $D_{T} \simeq 4.2 \hbar / m$ occurs well in the quantum critical region at $T \simeq 0.7 T_{F}$ (see Fig. 1). Hence, the quantum degenerate unitary Fermi gas is a nearly perfect fluid not only regarding momentum transport, but also for thermal transport.

With current sound propagation measurements in box traps reaching into the superfluid regime $[23,69]$, it will be particularly interesting to study critical scaling of the transport coefficients and observe the increase of $D_{T}$ shown in Fig. 1. This, as well as the related monotonic decrease of the Prandtl number indicated in Fig. 8, is due to the growing thermal conductivity associated with the crossover to classical critical fluctuations as expressed asymptotically in Eq. (2). For the sound diffusion $D_{\text {sound }}(T)$ (Fig. 1) both our quantum critical prediction and the experimental data indicate a monotonic decrease, while at even lower temperatures $T / T_{F} \lesssim 0.2$ an increase of $D_{\text {sound }}$ is again theoretically expected from critical fluctuations. In the strongly interacting 2D Fermi gas, the recently observed quantum scale anomaly [70] will have a large effect on the transport coefficients.

\section{ACKNOWLEDGMENTS}

We thank N. Defenu and M. W. Zwierlein for stimulating discussions, and M. W. Zwierlein for sharing experimental data. This work is supported by the Deutsche Forschungsgemeinschaft (DFG, German Research Foundation) via Collaborative Research Centre "SFB1225" (ISOQUANT) and under Germanys Excellence Strategy "EXC-2181/1-390900948" (the Heidelberg STRUCTURES Excellence Cluster).

\section{APPENDIX A: ENERGY CURRENT WARD IDENTITY}

Following Polyakov [45], energy conservation leads to the continuity equation $\partial_{t} \hat{\mathcal{H}}+\nabla \cdot \hat{j}^{E}=0$ between the energy density operator (Hamiltonian) and the energy current operator defined in Eq. (16). The expectation values of this operator equation together with two additional fermion operators then lead to the Ward identity for the full energy current vertex [cf. (22)]

$$
\begin{aligned}
\boldsymbol{q} \cdot & \mathcal{T}_{\sigma}^{E}(\boldsymbol{p}, \varepsilon, \boldsymbol{q}, \omega=0) \\
= & G_{\sigma}(\boldsymbol{p}, \varepsilon) G_{\sigma}(\boldsymbol{p}+\boldsymbol{q}, \varepsilon) \boldsymbol{q} \cdot \tilde{\mathcal{T}}_{\sigma}^{E}(\boldsymbol{p}, \varepsilon, \boldsymbol{q}, \omega=0) \\
= & \left(\varepsilon+\mu-\frac{\boldsymbol{q} \cdot(\boldsymbol{p}+\boldsymbol{q})}{2 m}\right) G_{\sigma}(\boldsymbol{p}+\boldsymbol{q}, \varepsilon) \\
& -\left(\varepsilon+\mu+\frac{\boldsymbol{q} \cdot \boldsymbol{p}}{2 m}\right) G_{\sigma}(\boldsymbol{p}, \varepsilon) \\
= & \boldsymbol{q} \cdot \frac{\partial G_{\sigma}[\mathbf{h}]}{\partial \mathbf{h}_{q}} .
\end{aligned}
$$

The amputated energy current vertex is then

$$
\begin{aligned}
& \boldsymbol{q} \cdot \tilde{\mathcal{T}}_{\sigma}^{E}(\boldsymbol{p}, \varepsilon, \boldsymbol{q}, \omega=0) \\
&=\left(\varepsilon+\mu-\frac{\boldsymbol{q} \cdot(\boldsymbol{p}+\boldsymbol{q})}{2 m}\right) G_{\sigma}^{-1}(\boldsymbol{p}, \varepsilon) \\
&-\left(\varepsilon+\mu+\frac{\boldsymbol{q} \cdot \boldsymbol{p}}{2 m}\right) G_{\sigma}^{-1}(\boldsymbol{p}+\boldsymbol{q}, \varepsilon) \\
&=-\boldsymbol{q} \cdot \frac{\partial G_{\sigma}^{-1}[\mathbf{h}]}{\partial \mathbf{h}_{q}}
\end{aligned}
$$

and by inserting the noninteracting Green's functions $G_{0}^{-1}(\boldsymbol{p}, \varepsilon)=\varepsilon-\varepsilon_{p}+\mu$ one finds the matrix elements of the bare energy current operator [cf. (17)]

$$
\tilde{\mathcal{T}}_{\sigma}^{E(0)}(\boldsymbol{p}, \boldsymbol{q})=j_{\sigma}^{E}(\boldsymbol{p}, \boldsymbol{q})=\frac{\boldsymbol{p}+\boldsymbol{q} / 2}{m} \times \frac{\boldsymbol{p} \cdot(\boldsymbol{p}+\boldsymbol{q})}{2 m} .
$$

In the two-channel model formulated in terms of both fermions and pairs, we have to introduce a new bosonic Ward identity beyond the one given by Polyakov [45]. We find for the full bosonic energy current vertex [cf. (22)]

$$
\begin{aligned}
& \boldsymbol{q} \cdot \mathcal{T}_{\text {pair }}^{E}(\boldsymbol{Q}, \Omega, \boldsymbol{q}, \omega=0) \\
&= \Gamma(\boldsymbol{Q}, \Omega) \Gamma(\boldsymbol{Q}+\boldsymbol{q}, \Omega) \boldsymbol{q} \cdot \tilde{\mathcal{T}}_{\mathrm{pair}}^{E}(\boldsymbol{Q}, \Omega, \boldsymbol{q}, \omega=0) \\
&=\left(\Omega+2 \mu+\frac{\boldsymbol{q} \cdot \boldsymbol{Q}}{2 m}\right) \Gamma(\boldsymbol{Q}+\boldsymbol{q}, \Omega) \\
&-\left(\Omega+2 \mu-\frac{\boldsymbol{q} \cdot(\boldsymbol{Q}+\boldsymbol{q})}{2 m}\right) \Gamma(\boldsymbol{Q}, \Omega) \\
&= \boldsymbol{q} \cdot \frac{\partial \Gamma[\mathbf{h}]}{\partial \mathbf{h}_{q}} .
\end{aligned}
$$

The amputated bosonic energy current vertex is then given by

$$
\begin{aligned}
& \boldsymbol{q} \cdot \tilde{\mathcal{T}}_{\text {pair }}^{E}(\boldsymbol{Q}, \Omega, \boldsymbol{q}, \omega=0) \\
&=\left(\Omega+2 \mu+\frac{\boldsymbol{q} \cdot \boldsymbol{Q}}{2 m}\right) \Gamma^{-1}(\boldsymbol{Q}, \Omega) \\
&-\left(\Omega+2 \mu-\frac{\boldsymbol{q} \cdot(\boldsymbol{Q}+\boldsymbol{q})}{2 m}\right) \Gamma^{-1}(\boldsymbol{Q}+\boldsymbol{q}, \Omega) \\
&=-\boldsymbol{q} \cdot \frac{\partial \Gamma^{-1}[\mathbf{h}]}{\partial \mathbf{h}_{q}} .
\end{aligned}
$$

When inserting the bare bosonic Green's function $\Gamma_{0}(Q, \Omega)=$ $\bar{g}(\Lambda)$, one recovers the matrix elements of the bosonic energy 
current operator [cf. (17)]

$$
\tilde{\mathcal{T}}_{\text {pair }}^{E(0)}(\boldsymbol{Q}, \boldsymbol{q})=j_{\text {pair }}^{E}(\boldsymbol{Q}, \boldsymbol{q})=\frac{\boldsymbol{Q}+\boldsymbol{q} / 2}{m \bar{g}(\Lambda)} .
$$

In the limit $\omega=0, \boldsymbol{q} \rightarrow 0$ the homogeneous Ward identities and current operators result that are given in the main text:

$$
\begin{aligned}
\mathcal{T}_{\sigma}^{E}(\boldsymbol{p}, \varepsilon) & =-\frac{\boldsymbol{p}}{m} G_{\sigma}(\boldsymbol{p}, \varepsilon)+(\varepsilon+\mu) \frac{\partial G_{\sigma}(\boldsymbol{p}, \varepsilon)}{\partial \boldsymbol{p}}, \\
\tilde{\mathcal{T}}_{\sigma}^{E}(\boldsymbol{p}, \varepsilon) & =-\frac{\boldsymbol{p}}{m} G_{\sigma}^{-1}(\boldsymbol{p}, \varepsilon)-(\varepsilon+\mu) \frac{\partial G_{\sigma}^{-1}(\boldsymbol{p}, \varepsilon)}{\partial \boldsymbol{p}}, \\
\tilde{\mathcal{T}}_{\sigma}^{E(0)}(\boldsymbol{p}, \varepsilon) & =\frac{\boldsymbol{p}}{m} \varepsilon_{p}, \\
\mathcal{T}_{\text {pair }}^{E}(\boldsymbol{Q}, \Omega) & =\frac{\boldsymbol{Q}}{m} \Gamma(\boldsymbol{Q}, \Omega)+(\Omega+2 \mu) \frac{\partial \Gamma(\boldsymbol{Q}, \Omega)}{\partial \boldsymbol{Q}}, \\
\tilde{\mathcal{T}}_{\text {pair }}^{E}(\boldsymbol{Q}, \Omega) & =\frac{\boldsymbol{Q}}{m} \Gamma^{-1}(\boldsymbol{Q}, \Omega)-(\Omega+2 \mu) \frac{\partial \Gamma^{-1}(\boldsymbol{Q}, \Omega)}{\partial \boldsymbol{Q}}, \\
\tilde{\mathcal{T}}_{\text {pair }}^{E(0)}(\boldsymbol{Q}, \Omega) & =\frac{\boldsymbol{Q}}{m} \frac{1}{\bar{g}(\Lambda)} .
\end{aligned}
$$

\section{APPENDIX B: UV ASYMPTOTICS OF CORRELATION FUNCTIONS}

The power-law tails typical for correlation functions in the zero-range limit arise from interaction effects, which are encoded in the fermionic self-energy $\Sigma\left(\mathbf{p} \rightarrow \infty, \tau \rightarrow 0^{-}\right)$ and in the pair propagator $\Gamma\left(\mathbf{Q} \rightarrow \infty, \tau \rightarrow 0^{-}\right)$. In order to formulate the Luttinger-Ward theory, it is more convenient to make use of the (anti)periodicity of (fermionic) bosonic correlation functions in imaginary time and to consider the limit $\tau \rightarrow \beta^{-}$instead of $\tau \rightarrow 0^{-}$. Within the self-consistent $T$-matrix approximation for the Luttinger-Ward grand potential the self-energy of unpolarized fermions is given by (henceforth $\hbar \equiv 1$ )

$$
\Sigma(\mathbf{p}, \tau)=-\int \frac{d^{3} Q}{(2 \pi)^{3}} \Gamma(\mathbf{Q}, \tau) G(\mathbf{Q}-\mathbf{p}, \beta-\tau) .
$$

In the limit of large momenta, both the Green's and the vertex functions approach their vacuum forms [71]

$$
\begin{aligned}
G(\mathbf{p} \rightarrow \infty, \tau) & \rightarrow G_{v}(\mathbf{p}, \tau) \simeq-e^{-\tau \varepsilon_{p}}, \\
\Gamma(\mathbf{Q} \rightarrow \infty, \tau) & \rightarrow \Gamma_{v}(\mathbf{Q}, \tau) \simeq-\frac{4 \sqrt{\pi}}{m^{3 / 2} \sqrt{\tau}} e^{-\tau \varepsilon_{Q} / 2} .
\end{aligned}
$$

Moreover, in the vacuum limit all diagrams vanish except for the particle-particle ladders which represent the exact $T$ matrix for two-particle scattering in quantum mechanics. Therefore, the Luttinger-Ward approach includes the correct exponents of the momentum tails. For $\tau \rightarrow \beta^{-}$ and $\mathbf{p} \rightarrow \infty$ we retain only the dominant contributions to the momentum integral which arise from the regions $|\mathbf{Q}| \ll|\mathbf{p}|$ and $|\mathbf{Q}-\mathbf{p}| \ll|\mathbf{p}|$. This allows one to expand the Green's function in the form

$$
G_{v}\left(\mathbf{Q}-\mathbf{p},(\beta-\tau) \rightarrow 0^{+}\right) \simeq-e^{-(\beta-\tau) \varepsilon_{p}}\left[1+(\beta-\tau)\left(\frac{\mathbf{Q} \cdot \mathbf{p}}{m}-\varepsilon_{Q}\right)+\frac{(\beta-\tau)^{2}}{2} \frac{(\mathbf{Q} \cdot \mathbf{p})^{2}}{m^{2}}+\cdots\right],
$$

and we obtain for the self-energy

$$
\begin{aligned}
\Sigma\left(\mathbf{p} \rightarrow \infty, \tau \rightarrow \beta^{-}\right) & \simeq e^{-(\beta-\tau) \varepsilon_{p}}\left[\Gamma\left(\mathbf{x}=\mathbf{0}, \tau \rightarrow \beta^{-}\right)+\left(\frac{4(\beta-\tau)^{2}}{3} \varepsilon_{p}-(\beta-\tau)\right) \int \frac{d^{3} Q}{(2 \pi)^{3}} \varepsilon_{Q} \Gamma\left(\mathbf{Q}, \tau \rightarrow \beta^{-}\right)\right], \\
& =e^{-(\beta-\tau) \varepsilon_{p}}\left[\Gamma\left(\mathbf{x}=\mathbf{0}, \tau \rightarrow \beta^{-}\right)+\left(\frac{4(\beta-\tau)^{2}}{3} \varepsilon_{p}-(\beta-\tau)\right)\left(-\frac{\nabla^{2}}{2 m}\right) \Gamma\left(\mathbf{x}, \tau \rightarrow \beta^{-}\right)_{\mathbf{x}=\mathbf{0}}\right] .
\end{aligned}
$$

Here, we have assumed that the momentum integral of the pair propagator is finite, which we show below in Eq. (B12). From our Luttinger-Ward data we find the behavior

$$
-\Gamma\left(\mathbf{x} \rightarrow \mathbf{0}, \tau \rightarrow \beta^{-}\right)=\mathcal{C} / m^{2}+\Gamma_{1}(\beta-\tau)+\Gamma_{3 / 2}(\beta-\tau)^{3 / 2}+\Gamma_{x^{2}} x^{2}+\cdots,
$$

where $\Gamma_{1,3 / 2, x^{2}}$ denote new coefficients while the leading order is determined by the Tan contact $\mathcal{C}$ according to Eq. (13). The anomalous power $(\beta-\tau)^{3 / 2}$ is generated by the self-consistent iteration but unbiased by the necessary analytic subtractions [34] which consider only contributions to the limit $\tau \rightarrow 0^{+}$. This result implies for the self-energy

$$
\begin{gathered}
\Sigma\left(\mathbf{p} \rightarrow \infty, \tau \rightarrow \beta^{-}\right) \rightarrow-e^{-(\beta-\tau) \varepsilon_{p}}\left[\mathcal{C}+\Gamma_{1}(\beta-\tau)+\Gamma_{3 / 2}(\beta-\tau)^{3 / 2}-\frac{\Gamma_{x^{2}}}{m}\left[4(\beta-\tau)^{2} \varepsilon_{p}-3(\beta-\tau)\right]\right], \\
\Sigma\left(\mathbf{p} \rightarrow \infty, \epsilon_{n}\right) \rightarrow \frac{\mathcal{C} / m^{2}}{i \epsilon_{n}+\varepsilon_{p}}+\frac{\Gamma_{1}+\frac{3}{m} \Gamma_{x^{2}}}{\left(i \epsilon_{n}+\varepsilon_{p}\right)^{2}}-\frac{\frac{8}{m} \Gamma_{x^{2}} \varepsilon_{p}}{\left(i \epsilon_{n}+\varepsilon_{p}\right)^{3}}+\frac{3 \sqrt{\pi} \Gamma_{3 / 2}}{4\left(i \epsilon_{n}+\varepsilon_{p}\right)^{5 / 2}} .
\end{gathered}
$$

Here, the second line is obtained from the first one by Fourier transform to Matsubara frequencies. Using the Dyson equation $G^{-1}\left(\mathbf{p}, \epsilon_{n}\right)=G_{0}^{-1}\left(\mathbf{p}, \epsilon_{n}\right)-\Sigma\left(\mathbf{p}, \epsilon_{n}\right)$ one can determine the asymptotic power laws of the momentum distribution

$$
n(\mathbf{p} \rightarrow \infty)=\frac{1}{\beta} \sum_{n} \frac{\Sigma\left(\mathbf{p} \rightarrow \infty, \epsilon_{n}\right)}{\left(i \epsilon_{n}-\varepsilon_{p}\right)^{2}}=\frac{\mathcal{C}}{p^{4}}+\frac{\Gamma_{1}-\frac{3}{m} \Gamma_{x^{2}}}{4 \varepsilon_{p}^{3}}+\frac{15 \sqrt{2 \pi} \Gamma_{3 / 2}}{128 \varepsilon_{p}^{7 / 2}} \cdots,
$$

which is indeed of the form (29) stated in the main text. 
We turn now to the UV behavior of the pair propagator $\Gamma\left(\mathbf{Q} \rightarrow \infty, \tau \rightarrow \beta^{-}\right)$. In the ladder approximation it can be expressed via the Bethe-Salpeter equation

$$
\Gamma\left(\mathbf{Q}, \Omega_{n}\right)=\frac{1}{1 / g+M_{p p}\left(\mathbf{Q}, \Omega_{n}\right)},
$$

where $M_{p p}$ denotes the renormalized particle-particle bubble

$$
M_{p p}(\mathbf{Q}, \tau)=\int \frac{d^{3} p}{(2 \pi)^{3}} G(\mathbf{p}, \tau) G(\mathbf{Q}-\mathbf{p}, \tau) .
$$

However, the cancellation of divergent terms in the zerorange limit affects only the behavior $\tau \rightarrow 0^{+}$and needs not to be taken further into account. Employing analogous arguments that led from the convolution (B1) to the result (B4) while using the asymptotic form [71] $G(\mathbf{p} \rightarrow \infty, \tau \rightarrow$ $\left.\beta^{-}\right) \rightarrow-\left(\mathcal{C} / p^{4}\right) \exp \left[-\varepsilon_{p}(\beta-\tau)\right]$, we obtain the limiting behavior

$$
M_{p p}^{\beta}(\mathbf{Q}, \tau)=M_{p p}\left(\mathbf{Q} \rightarrow \infty, \tau \rightarrow \beta^{-}\right) \rightarrow \frac{n \mathcal{C}}{Q^{4}} e^{-\varepsilon_{Q}(\beta-\tau)},
$$

where we have inserted the total density $n=-2 G(\mathbf{x}=$ $\left.0, \beta^{-}\right)$. Transforming this to frequency space yields

$$
M_{p p}^{\beta}\left(\mathbf{Q}, \Omega_{n}\right)=\frac{n \mathcal{C}}{Q^{4}} \frac{1}{\varepsilon_{Q}+i \Omega_{n}},
$$

which combines with the leading contribution in the vacuum limit $M_{p p}\left(\mathbf{Q}, \Omega_{n} \rightarrow \infty\right) \rightarrow-m^{3 / 2} \sqrt{\varepsilon_{Q}-2 i \Omega_{n}} /\left(2^{5 / 2} \pi\right)$ to yield the pair momentum distribution (of dimension wave number due to the anomalous dimension of the contact operator)

$$
n_{\text {pair }}(Q)=-m^{2} \Gamma\left(\mathbf{Q}, \tau \rightarrow \beta^{-}\right)=\frac{64 \pi^{2}}{3} \frac{n \mathcal{C}}{Q^{6}}+\cdots .
$$

\section{APPENDIX C: VARIATIONAL KINETIC THEORY}

In this Appendix we explain how to evaluate the variational bound on the transport scattering rate (33) in a larger basis set. Specifically for the shear viscosity, $X_{p}=p_{x} p_{y} / m$ denotes the shear perturbation and $U_{p}$ has the same quadrupole symmetry as $X_{p}$, hence it differs from $X_{p}$ only by a spherically symmetric function of $p^{2}$. One can expand $U_{\boldsymbol{p}}=\sum_{i} c_{i} U_{i}(\boldsymbol{p})$ in orthogonal (but not necessarily normalized) basis functions $U_{i}$ with $\left(U_{i}, U_{j}\right)=u_{i} \delta_{i j}$. A particularly convenient choice is setting $U_{1}(\boldsymbol{p})=X(\boldsymbol{p})$ and finding orthogonal $U_{i}, i>1$, by the Gram-Schmidt method, which simplifies Eq. (33) to

$$
\tau^{-1}=\min _{U(p)} \frac{(U, H U)}{c_{1}^{2}(X, X)} .
$$

The collision integral is normalized by $(X, X)$, which in the case of the shear viscosity is proportional to the pressure of the ideal Fermi gas, $(X, X)=-\lambda_{T}^{-3} T^{2} \mathrm{Li}_{5 / 2}\left(-e^{\beta \mu}\right)$. Denoting the matrix elements of the positive linear operator $H$ as $H_{i j}=$ $\left(U_{i}, H U_{j}\right)$, the stationarity of $\tau^{-1}$ with respect to variations in $U$ requires $\tau^{-1} \delta_{i 1} c_{1}(X, X)=\sum_{j} H_{i j} c_{j}$. The minimum principle for $\tau^{-1}$ then implies that each minimization within a finite subspace of $U_{i}$, for $i=1, \ldots, M$, provides an upper bound on the true value of $\tau^{-1}$, which can be successively improved (lowered) by increasing $M$. Equivalently, this can be expressed

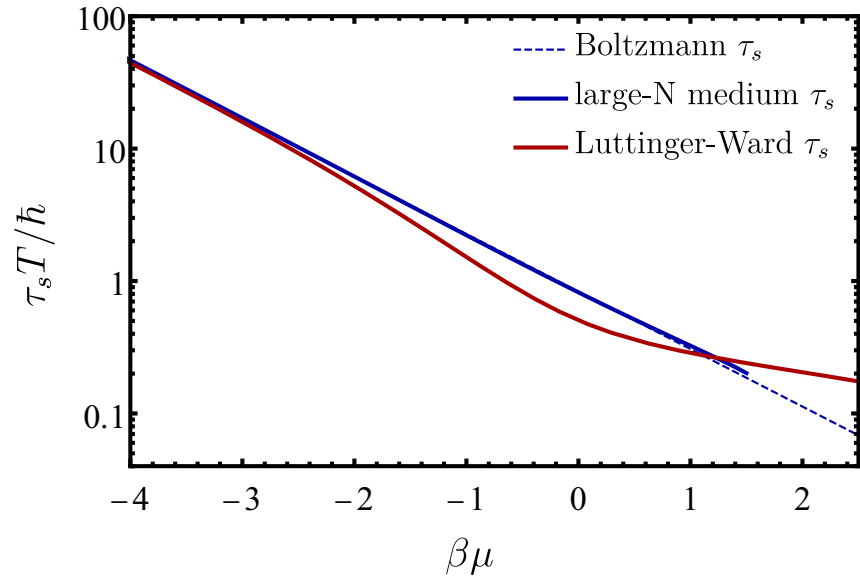

FIG. 9. Spin scattering times $\tau_{s}$ from Boltzmann (dashed) and large- $N$ calculations (blue) coincide and agree well with LuttingerWard results (red) in the quantum critical regime.

as a lower bound on the scattering time

$$
\tau \geqslant\left(H^{-1}\right)_{11}(X, X),
$$

in terms of the $(1,1)$ element of the inverse matrix of $H_{i j}$. Results for the viscous scattering time $\tau_{\eta}$ in the unitary Fermi gas are shown in Fig. 5 in the main text. The surprising observation for the viscous scattering time $\tau_{\eta}$ at unitarity is that it has nearly the same value both with a Boltzmann distribution and with a Fermi-Dirac distribution, but only if a full variational basis set beyond the first basis function $U_{1}$ is used.

Analogously, a similar observation is made for the heat conductivity with driving term $X_{p}=\left(\varepsilon_{p}-w\right) \frac{p}{m}$. Again, we choose a set of variational basis functions $U_{i}(\boldsymbol{p})=p^{2(i-1)} X_{p}$, for $i=1, \ldots, M$ [34], and find that the thermal transport scattering time converges rapidly already with the first three basis functions, but differs markedly from the result with only the first basis function $U_{1}$. Finally, also for spin diffusion we compute the transport scattering time with more than one basis function in the quantum degenerate regime. The spin diffusivity $D_{s}$ is defined via the Einstein relation in terms of

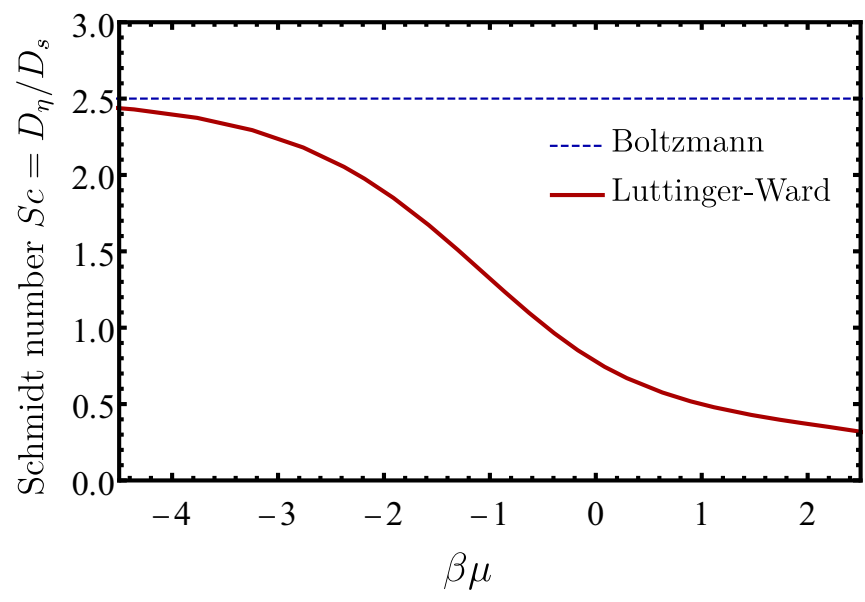

FIG. 10. Schmidt number $\mathrm{Sc}=D_{\eta} / D_{s}$ in the quantum critical regime, combining quantum critical scattering times from our large$N$ calculation with the Luttinger-Ward equation of state. The dashed line denotes the Boltzmann limit $\mathrm{Sc}=\frac{5}{2}$. 
spin conductivity $\sigma_{s}$ and spin susceptibility $\chi_{s}[20]$ :

$$
D_{s}=\frac{\sigma_{s}}{\chi_{s}}=\frac{n \tau_{s}}{m \chi_{s}}
$$

The spin scattering time $\tau_{s}$ shown in Fig. 9 also exhibits the quantum critical scaling that we observed already for shear and thermal transport: the medium scattering time is, within our numerical resolution, identical to the Boltzmann scattering time $\tau_{s} T / \hbar=\frac{3 \pi}{8 \sqrt{2}} e^{-\beta \mu}$. The quantum critical scattering time is now combined with the Luttinger-Ward equation of state for density $n$ and spin susceptibility $\chi_{s}$ to obtain the spin diffusivity $D_{s}$. Now, the Schmidt number [62]

$$
\mathrm{Sc}=\frac{D_{\eta}}{D_{s}}=\frac{(p / m n) \tau_{\eta}}{\left(n / m \chi_{s}\right) \tau_{s}}=\frac{p \chi_{s}}{n^{2}} \times \frac{\tau_{\eta}}{\tau_{s}}
$$

is defined as the dimensionless transport ratio of shear and spin diffusion and characterizes the relative importance of momentum and spin relaxation. As shown in Fig. 10, the Schmidt number starts from a value of $\mathrm{Sc}=\tau_{\eta} / \tau_{s}=\frac{5}{2}$ in the high-temperature limit and drops to around 0.3 near $T_{c}$, indicating that momentum diffusion is suppressed by a factor of almost 10 relative to spin diffusion. This is physically expected because viscosity is carried both by single fermions and pairs and therefore strongly affected by pair fluctuations near $T_{c}$, whereas pairs carry no spin current.
[1] V. J. Emery and S. A. Kivelson, Superconductivity in Bad Metals, Phys. Rev. Lett. 74, 3253 (1995).

[2] J. A. N. Bruin, H. Sakai, R. S. Perry, and A. P. Mackenzie, Similarity of scattering rates in metals showing $T$-linear resistivity, Science 339, 804 (2013).

[3] B. Keimer, S. A. Kivelson, M. R. Norman, S. Uchida, and J. Zaanen, From quantum matter to high-temperature superconductivity in copper oxides, Nature (London) 518, 179 (2015).

[4] R. Mahajan, M. Barkeshli, and S. A. Hartnoll, Non-Fermi liquids and the Wiedemann-Franz law, Phys. Rev. B 88, 125107 (2013).

[5] S. Sachdev, Quantum Phase Transitions (Cambridge University Press, Cambridge, 2011).

[6] S. A. Hartnoll, A. Lucas, and S. Sachdev, Holographic Quantum Matter (MIT Press, Cambridge, MA, 2018).

[7] A. Lucas, Operator Size at Finite Temperature and Planckian Bounds on Quantum Dynamics, Phys. Rev. Lett. 122, 216601 (2019).

[8] J. Zhang, E. D. Kountz, E. M. Levenson-Falk, D. Song, R. L. Greene, and A. Kapitulnik, Thermal diffusivity above the Mott-Ioffe-Regel limit, Phys. Rev. B 100, 241114(R) (2019).

[9] The BCS-BEC Crossover and the Unitary Fermi Gas, Lecture Notes in Physics Vol. 836, edited by W. Zwerger (Springer, Berlin, 2012).

[10] W. Zwerger, Strongly interacting Fermi gases, in Quantum matter at ultralow temperatures, Proceedings of the International School of Physics "Enrico Fermi," Course 191, Varenna 2014, edited by M. Inguscio, W. Ketterle, S. Stringari, and G. Roati (IOS Press, Amsterdam, 2016), pp. 63-141.

[11] P. Nikolić and S. Sachdev, Renormalization-group fixed points, universal phase diagram, and $1 / N$ expansion for quantum liquids with interactions near the unitarity limit, Phys. Rev. A 75, 033608 (2007).

[12] S. Sachdev, Dilute Fermi and Bose Gases, in The BCS-BEC Crossover and the Unitary Fermi Gas, edited by W. Zwerger (Springer, Berlin, 2012), Chap. 8, p. 277.

[13] Y. Nishida and D. T. Son, Nonrelativistic conformal field theories, Phys. Rev. D 76, 086004 (2007).

[14] Y. Nishida and D. T. Son, Unitary Fermi gas, $\varepsilon$ expansion, and nonrelativistic conformal field theories, in The BCS-BEC Crossover and the Unitary Fermi Gas, edited by W. Zwerger (Springer, Berlin, 2012), Chap. 7, p. 233.
[15] P. K. Kovtun, D. T. Son, and A. O. Starinets, Viscosity in Strongly Interacting Quantum Field Theories from Black Hole Physics, Phys. Rev. Lett. 94, 111601 (2005).

[16] T. Enss, R. Haussmann, and W. Zwerger, Viscosity and scale invariance in the unitary Fermi gas, Ann. Phys. (NY) 326, 770 (2011).

[17] J. A. Joseph, E. Elliott, and J. E. Thomas, Shear Viscosity of a Unitary Fermi Gas Near the Superfluid Phase Transition, Phys. Rev. Lett. 115, 020401 (2015).

[18] M. Bluhm, J. Hou, and T. Schäfer, Determination of the Density and Temperature Dependence of the Shear Viscosity of a Unitary Fermi Gas Based on Hydrodynamic Flow, Phys. Rev. Lett. 119, 065302 (2017).

[19] A. Sommer, M. Ku, G. Roati, and M. W. Zwierlein, Universal spin transport in a strongly interacting Fermi gas, Nature (London) 472, 201 (2011).

[20] T. Enss and R. Haussmann, Quantum Mechanical Limitations to Spin Transport in the Unitary Fermi Gas, Phys. Rev. Lett. 109, 195303 (2012).

[21] S. Trotzky, S. Beattie, C. Luciuk, S. Smale, A. B. Bardon, T. Enss, E. Taylor, S. Zhang, and J. H. Thywissen, Observation of the Leggett-Rice Effect in a Unitary Fermi Gas, Phys. Rev. Lett. 114, 015301 (2015).

[22] G. Valtolina, F. Scazza, A. Amico, A. Burchianti, A. Recati, T. Enss, M. Inguscio, M. Zaccanti, and G. Roati, Exploring the ferromagnetic behaviour of a repulsive Fermi gas through spin dynamics, Nat. Phys. 13, 704 (2017).

[23] P. B. Patel, Z. Yan, B. Mukherjee, R. J. Fletcher, J. Struck, and M. W. Zwierlein, Universal sound diffusion in a strongly interacting Fermi gas, arXiv:1909.02555.

[24] B. Mukherjee, Z. Yan, P. B. Patel, Z. Hadzibabic, T. Yefsah, J. Struck, and M. W. Zwierlein, Homogeneous Atomic Fermi Gases, Phys. Rev. Lett. 118, 123401 (2017).

[25] K. Hueck, N. Luick, L. Sobirey, J. Siegl, T. Lompe, and H. Moritz, Two-Dimensional Homogeneous Fermi Gases, Phys. Rev. Lett. 120, 060402 (2018).

[26] H. Hu, P. Zou, and X.-J. Liu, Low-momentum dynamic structure factor of a strongly interacting Fermi gas at finite temperature: A two-fluid hydrodynamic description, Phys. Rev. A 97, 023615 (2018).

[27] P. Zhang and Z. Yu, Energy-absorption spectroscopy of unitary Fermi gases in a uniform potential, Phys. Rev. A 97, 041601(R) (2018). 
[28] L. Baird, X. Wang, S. Roof, and J. E. Thomas, Measuring the Hydrodynamic Linear Response of a Unitary Fermi Gas, Phys. Rev. Lett. 123, 160402 (2019).

[29] C. C. N. Kuhn, S. Hoinka, I. Herrera, P. Dyke, J. J. Kinnunen, G. M. Bruun, and C. J. Vale, High-Frequency Sound in a Unitary Fermi Gas, Phys. Rev. Lett. 124, 150401 (2020).

[30] D. Forster, Hydrodynamic Fluctuations, Broken Symmetry, and Correlation Functions (WA Benjamin, Reading, 1975).

[31] M. Braby, J. Chao, and T. Schäfer, Thermal conductivity and sound attenuation in dilute atomic Fermi gases, Phys. Rev. A 82, 033619 (2010).

[32] R. Haussmann, W. Rantner, S. Cerrito, and W. Zwerger, Thermodynamics of the BCS-BEC crossover, Phys. Rev. A 75, 023610 (2007).

[33] B. Frank, J. Lang, and W. Zwerger, Universal phase diagram and scaling functions of imbalanced Fermi gases, J. Exp. Theor. Phys. 127, 812 (2018).

[34] B. Frank, Thermodynamics and transport in fermi gases near unitarity, Ph.D. thesis, Technische Universität München, 2018.

[35] C. J. Pethick and H. Smith, Bose Einstein Condensation in Dilute Gases, 2nd ed. (Cambridge University Press, Cambridge, 2008).

[36] T. Enss, Quantum critical transport in the unitary Fermi gas, Phys. Rev. A 86, 013616 (2012).

[37] M. J. H. Ku, A. T. Sommer, L. W. Cheuk, and M. W. Zwierlein, Revealing the Superfluid Lambda Transition in the Universal Thermodynamics of a Unitary Fermi Gas, Science 335, 563 (2012).

[38] G. Baym, J.-P. Blaizot, and J. Zinn-Justin, The transition temperature of the dilute interacting Bose gas for $N$ internal states, Europhys. Lett. 49, 150 (2000).

[39] T. Debelhoir and N. Dupuis, Critical region of the superfluid transition in the BCS-BEC crossover, Phys. Rev. A 93, 023642 (2016).

[40] P. C. Hohenberg and B. I. Halperin, Theory of dynamic critical phenomena, Rev. Mod. Phys. 49, 435 (1977).

[41] R. A. Ferrell, N. Menyhard, H. Schmidt, F. Schwabl, and P. Szépfalusy, Dispersion in Second Sound and Anomalous Heat Conduction at the Lambda Point of Liquid Helium, Phys. Rev. Lett. 18, 891 (1967).

[42] L. P. Kadanoff and P. C. Martin, Hydrodynamic equations and correlation functions, Ann. Phys. (NY) 24, 419 (1963).

[43] W. Götze and P. Wölfle, Homogeneous dynamical conductivity of simple metals, Phys. Rev. B 6, 1226 (1972).

[44] S. A. Hartnoll, R. Mahajan, M. Punk, and S. Sachdev, Transport near the Ising-nematic quantum critical point of metals in two dimensions, Phys. Rev. B 89, 155130 (2014).

[45] A. M. Polyakov, Nonequilibrium processes in the critical region, J. Exp. Theor. Phys. 30, 1164 (1970) [Zh. Eksp. Teor. Fiz. 57, 2144 (1969)].

[46] G. Zürn, T. Lompe, A. N. Wenz, S. Jochim, P. S. Julienne, and J. M. Hutson, Precise Characterization of ${ }^{6} \mathrm{Li}$ Feshbach Resonances using Trap-Sideband-Resolved RF Spectroscopy of Weakly Bound Molecules, Phys. Rev. Lett. 110, 135301 (2013).

[47] S. Tan, Energetics of a strongly correlated Fermi gas, Ann. Phys. (NY) 323, 2952 (2008).

[48] B. Mukherjee, P. B. Patel, Z. Yan, R. J. Fletcher, J. Struck, and M. W. Zwierlein, Spectral Response and Contact of the Unitary Fermi Gas, Phys. Rev. Lett. 122, 203402 (2019).
[49] R. Haussmann, M. Punk, and W. Zwerger, Spectral functions and $\mathrm{rf}$ response of ultracold fermionic atoms, Phys. Rev. A 80 063612 (2009).

[50] B. S. Shastry, Sum rule for thermal conductivity and dynamical thermal transport coefficients in condensed matter, Phys. Rev. B 73, 085117 (2006).

[51] A. Altland and B. Simons, Condensed Matter Field Theory (Cambridge University Press, Cambridge, 2006).

[52] K. Fujii and Y. Nishida, Hydrodynamics with space-timedependent scattering length, Phys. Rev. A 98, 063634 (2018).

[53] E. Taylor and M. Randeria, Viscosity of strongly interacting quantum fluids: spectral functions and sum rules, Phys. Rev. A 81, 053610 (2010).

[54] T. Enss, Bulk Viscosity and Contact Correlations in Attractive Fermi Gases, Phys. Rev. Lett. 123, 205301 (2019).

[55] T. Enss, Shear viscosity and spin sum rules in strongly interacting Fermi gases, Eur. Phys. J.: Spec. Top. 217, 169 (2013).

[56] E. Braaten and L. Platter, Exact Relations for a Strongly Interacting Fermi Gas from the Operator Product Expansion, Phys. Rev. Lett. 100, 205301 (2008).

[57] F. Werner and Y. Castin, General relations for quantum gases in two and three dimensions. Two-component fermions, Phys. Rev. A 86, 013626 (2012).

[58] Y. Nishida, Viscosity spectral functions of resonating fermions in the quantum virial expansion, Ann. Phys. (NY) 410, 167949 (2019).

[59] J. Hofmann, High-temperature expansion of the viscosity in interacting quantum gases, Phys. Rev. A 101, 013620 (2020).

[60] G. Haines and A. Jones, Logarithmic fourier transformation, Geophys. J. R. Astron. Soc. 92, 171 (1988).

[61] J. Lang and B. Frank, Fast logarithmic Fourier-Laplace transform of nonintegrable functions, Phys. Rev. E 100, 053302 (2019).

[62] H. Smith and H. H. Jensen, Transport Phenomena (Oxford University Press, Oxford, UK, 1989).

[63] T. Schäfer, Second-order fluid dynamics for the unitary Fermi gas from kinetic theory, Phys. Rev. A 90, 043633 (2014).

[64] P. Massignan, G. M. Bruun, and H. Smith, Viscous relaxation and collective oscillations in a trapped Fermi gas near the unitarity limit, Phys. Rev. A 71, 033607 (2005).

[65] G. M. Bruun and H. Smith, Shear viscosity and damping for a Fermi gas in the unitarity limit, Phys. Rev. A 75, 043612 (2007).

[66] G. M. Bruun, Feshbach Resonances and Medium Effects in Ultracold Atomic Gases, Few-Body Syst. 45, 227 (2009).

[67] T. Enss and J. H. Thywissen, Universal Spin Transport and Quantum Bounds for Unitary Fermions, Annu. Rev. Condens. Matter Phys. 10, 85 (2019).

[68] M. Rangamani, S. F. Ross, D. T. Son, and E. G. Thompson, Conformal non-relativistic hydrodynamics from gravity, J. High Energy Phys. 01 (2009) 075.

[69] M. Bohlen, L. Sobirey, N. Luick, H. Biss, T. Enss, T. Lompe, and $\mathrm{H}$. Moritz, Sound propagation and quantum limited damping in a two-dimensional Fermi gas, arXiv:2003.02713 [Phys. Rev. Lett. (to be published)].

[70] P. A. Murthy, N. Defenu, L. Bayha, M. Holten, P. M. Preiss, T. Enss, and S. Jochim, Quantum scale anomaly and spatial coherence in a 2D Fermi superfluid, Science 365, 268 (2019).

[71] K. Van Houcke, F. Werner, T. Ohgoe, N. V. Prokof'ev, and B. V. Svistunov, Diagrammatic Monte Carlo algorithm for the resonant Fermi gas, Phys. Rev. B 99, 035140 (2019). 